\title{
Floristic Surveys of Saguaro National Park Protected Natural Areas
}

William L. Halvorson and Brooke S. Gebow, editors

Technical Report No. 68

United States Geological Survey

Sonoran Desert Field Station

The University of Arizona $\bullet$ Tucson, Arizona 


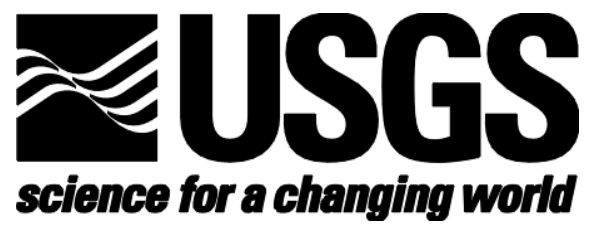

\section{USGS Sonoran Desert Field Station The University of Arizona, Tucson}

The Sonoran Desert Field Station (SDFS) at The University of Arizona is a unit of the USGS Western Ecological Research Center (WERC). It was originally established as a National Park Service Cooperative Park Studies Unit (CPSU) in 1973 with a research staff and ties to The University of Arizona. Transferred to the USGS Biological Resources Division in 1996, the SDFS continues the CPSU mission of providing scientific data (1) to assist U.S. Department of Interior land management agencies within Arizona and (2) to foster cooperation among all parties overseeing sensitive natural and cultural resources in the region. It also is charged with making its data resources and researchers available to the interested public. Seventeen such field stations in California, Arizona, and Nevada carry out WERC's work.

The SDFS provides a multi-disciplinary approach to studies in natural and cultural sciences. Principal cooperators include the School of Renewable Natural Resources and the Department of Ecology and Evolutionary Biology at The University of Arizona. Unit scientists also hold faculty or research associate appointments at the university.

The Technical Report series distributes information relevant to high priority regional resource management needs. The series presents detailed accounts of study design, methods, results, and applications possibly not accommodated in the formal scientific literature. Technical Reports follow SDFS guidelines and are subject to peer review and editing. Publications may contain findings that are preliminary but have immediate use to field workers in the region. Print runs are small, and distribution is generally limited to parties with a direct involvement in report topics.

\section{ARIZONA.}

TUCSON ARIZONA 


\title{
Floristic Surveys of Saguaro National Park Protected Natural Areas
}

\author{
William L. Halvorson \\ Brooke S. Gebow \\ Editors
}

Technical Report No. 68

October 2000

\author{
United States Geological Survey \\ Western Ecological Research Center \\ Sonoran Desert Field Station \\ and \\ School of Renewable Natural Resources \\ 125 Biological Sciences East \\ The University of Arizona \\ Tucson, Arizona 85721
}




\section{Editors}

William L. Halvorson

USGS Sonoran Desert Field Station

The University of Arizona

125 Biological Sciences East

Tucson, Arizona 85721

Brooke S. Gebow

School of Renewable Natural Resources

The University of Arizona

125 Biological Sciences East

Tucson, Arizona 85721

\section{USGS Sonoran Desert Field Station Personnel}

William L. Halvorson, Research Ecologist

Cecil R. Schwalbe, Ecologist

Peter S. Bennett, Ecologist

Michael R. Kunzmann, Ecologist

Brooke S. Gebow, Editor

Sandra Mosolf, Administrative Assistant

Brenda Carbajal, Secretary

(520) 670-6885; fax (520) 670-5001

As the nation's largest water, earth, and biological science and civilian mapping agency, the USGS works in cooperation with more than 200 organizations across the country to provide reliable, impartial, scientific information to resource managers, planners, and other customers. This information is gathered in every state by USGS scientists to minimize loss of life and property from natural disasters, contribute to sound economic and physical development of the nation's natural resources, and enhance the quality of life by monitoring water, biological, energy, and mineral resources.

Reports in this series are produced in limited quantities. As long as the supply lasts, copies may be obtained from USGS Sonoran Desert Field Station, 125 Biological Sciences East, The University of Arizona, Tucson, AZ 85721.

This report was printed on recycled paper. 


\section{Contents}

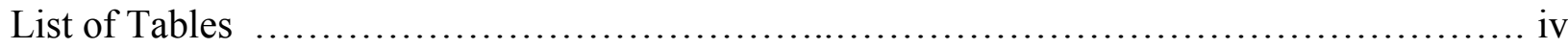

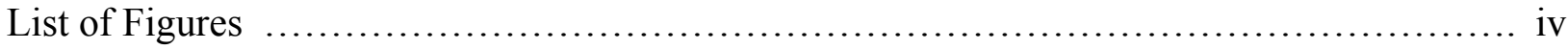

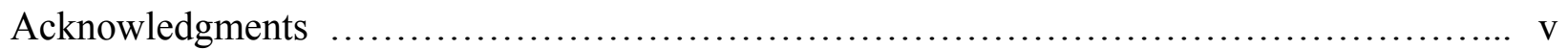

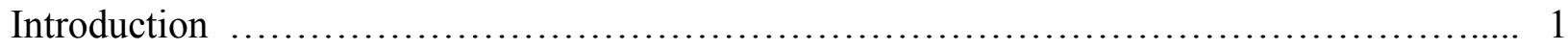

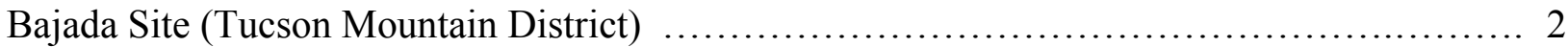

Methods …........................................................................ 2

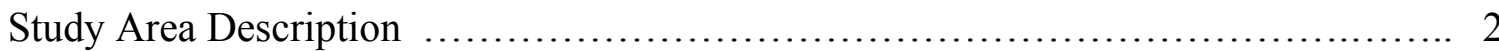

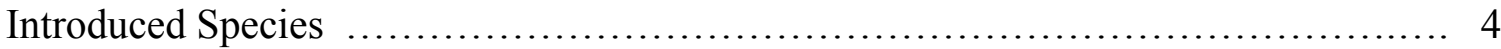

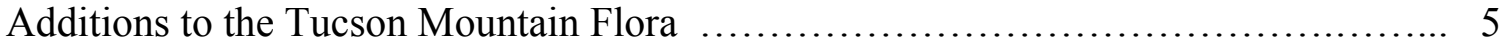

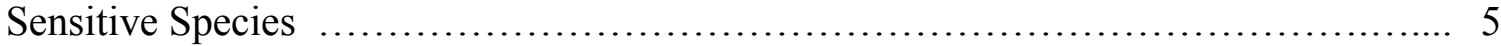

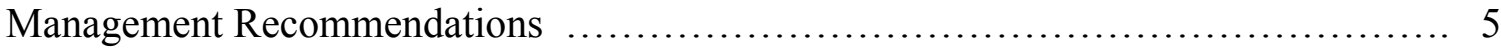

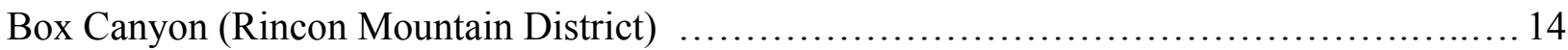

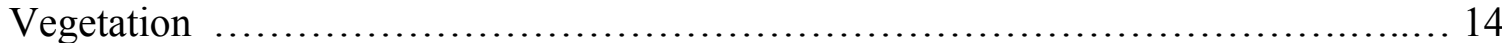

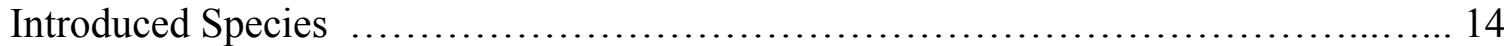

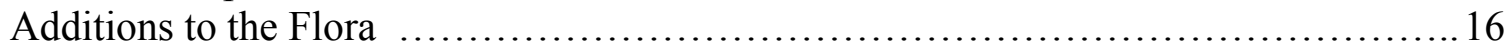

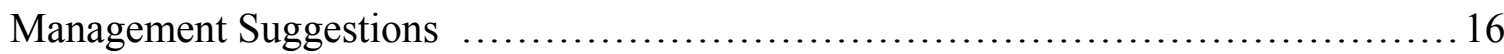

Chimenea and Madrona Canyons (Rincon Mountain District) …...........................25

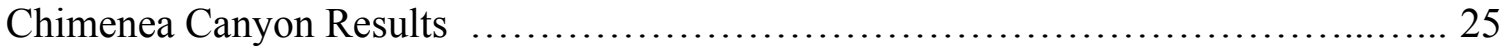

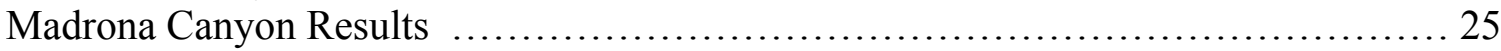

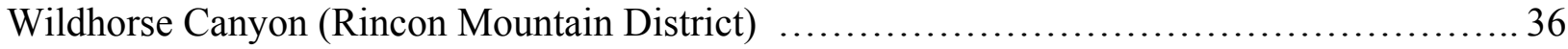

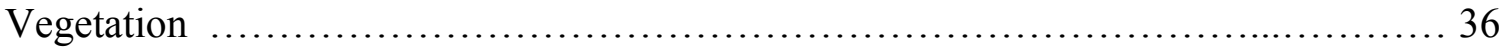

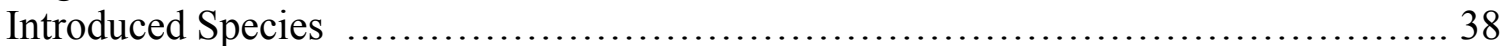

Additions to the Flora ................................................................ 39

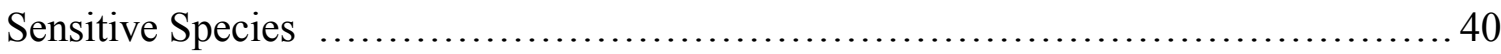

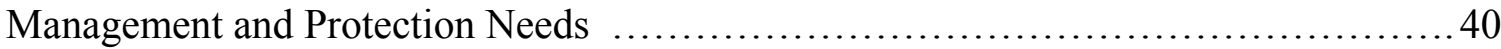

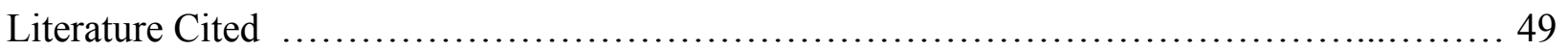




\section{Figures}

Figure 1. Bajada Site Plant Survey Location within the Tucson Mountain District of Saguaro National Park

Figure 2. Plant Survey Locations within the Rincon Mountain

District of Saguaro National Park 15

\section{Tables}

Table 1. Bajada Site Checklist of Vascular Plants f................................... 7

Table 2. Box Canyon Checklist of Vascular Plants $\quad$.................................... 17

Table 3. Chimenea Canyon Checklist of Vascular Plants ….......................... 26

Table 4. Madrona Canyon Checklist of Vascular Plants …............................. 34

Table 5. Wildhorse Canyon Checklist of Vascular Plants .............................. 42 


\section{Acknowledgments}

The editors acknowledge the efforts of the many people it took to create this report. Our thanks go to the following section authors, plant surveyors, Saguaro National Park staff, and reviewers:

\section{Section Authors}

Rebecca Van Devender (Bajada)

Mark Fishbein, Victor Steinman, and Amy Johnson (Box Canyon)

Renée Rondeau and Rebecca Van Devender (Wildhorse Canyon)

\section{Plant Surveyors}

The section authors were assisted with the plant surveys by George Ferguson, Michelle Hawkes, Kathy Hiett, Rachel Levin, Shelley McMahon, Jan Miller, Tricia Roller, and Don Swann.

\section{Saguaro National Park Staff}

Meg Weesner, Chief of Science and Resource Management, facilitated completion of the project and Pam Anning produced the maps (Figures 1 and 2).

\section{Reviewers}

Patty Guertin, USGS Sonoran Desert Field Station, brought plant names up to date, and Cecily Gill and Lynn Kaufman from Tucson Botanical Gardens reviewed the manuscript and proofread plant names. 


\section{Introduction}

This report contains four floristic surveys from Saguaro National Park near Tucson, Arizona. The surveys were conducted between 1992 and 1995 after the park gave the four sites Protected Natural Area (PNA) status. PNA status signifies that areas contain important geological or ecological values. With the designations in place, the park tries to "zone" for uses that minimize human interference in these fragile or unique areas. Ecologically important PNAs such as those addressed in this report serve as:

1. Baseline areas against which the effects of human activities in similar areas can be measured

2. Sites for the study of natural processes in undisturbed ecosystems

3. Gene pool reserves for plant and animals species, particularly rare and endangered taxa

Three of the sites lie within the Rincon Mountain District of the park: Box Canyon, Madrona/ Chiminea Canyon, and Wildhorse Canyon. The fourth is within the jurisdiction of the Tucson Mountain District and is called the "Bajada" site in this report. The Bajada survey concludes that the area is floristically diverse and interesting, but no more so than other low bajada areas within the west unit. Given the lack of trails in the area, the PNA status is probably not necessary.

Each plant list is based on selected transects within the PNA. Surveyors walked the transects and recorded all species encountered. They collected two sets of voucher specimens. The first set contains samples of all species for the site, and in each case these specimens were deposited in the herbarium at the University of Arizona. The second set consists of plants previously unrecorded in Saguaro National Park. Those specimens now reside in the park herbarium. Plant names in this report follow Kartesz (1994).

As a group, the studies provide valuable detail about the resources within Saguaro National Park. The plant lists also form a snapshot of the park flora in the 1990s. Surveyors noted exotic and rare species and also found plants unreported from both the Rincon and Tucson Mountain Districts of the park. This publication brings the information in the four studies together into a single volume for use by other workers. 


\title{
Bajada Site (Tucson Mountain District)
}

\author{
By Rebecca Van Devender
}

Sections 21 and 28 of T13S, R11E in the Tucson Mountain Unit of Saguaro National Park (Figure 1) were designated as a Protected Natural Area in the General Management Plan (NPS 1988 ) and, as such, were given extra protection from disturbance and over-visitation within the park. This site was given the name "Bajada." The area is $520 \mathrm{ha}\left(2 \mathrm{mi}^{2}\right)$ of gently sloping bajada west of Sandario Road that is for the most part a rich paloverde-saguaro-ironwood desertscrub with a strong admixture of creosotebush (Larrea tridentata), triangleleaf bursage (Ambrosia deltoidea), ocotillo (Fouquieria splendens) and various cacti. Sections 21 and 28 are adjacent and bordered on all four sides by roads or human habitations (Figure 1). A total of 197 species in 44 families was found in the area (see Table 1 at the end of the chapter).

\section{Methods}

Surveys were conducted in both spring and summer of 1992 to compile a baseline inventory of the flora. The site was visited on April 7 and again on August 24 and 25 to obtain a thorough sampling of herbaceous vegetation, both annual and perennial, which might be apparent only in spring or summer.

In the spring, nine east-west transects were walked in an effort to cover uniformly as much of the available habitats as possible. In addition to the fairly flat bajada, which comprises the majority of the area, all four major east-west washes and areas along the perimeter on all four sides were sampled. The summer survey of eight east-west line transects emphasized the major washes where most of the summer herbs were concentrated. Little diversity was found on the open flats or on the perimeter.

\section{Study Area Description}

Sections 21 and 28 have fairly uniform topography with less than $46 \mathrm{~m}$ (150 ft) change in elevation. Elevation gradually decreases from $720 \mathrm{~m}(2360 \mathrm{ft})$ in the extreme southeast corner of $\mathrm{S} 28$ to $670 \mathrm{~m}(2200 \mathrm{ft})$ in the extreme northwest corner of S21. There is a gradual drop in elevation as well from east to west in both sections. Soil texture also changes from east to west and from south to north on the gently sloping bajada toward Avra Valley. Bedrock of the Tucson Mountains lies just east of Sandario Road in the NW1/4 of S27. Coarse and gravelly soils on the upper bajada grade into fine-grained silts and clays in the valley bottom. Changes in soils and, probably to a lesser extent, the decrease in elevation, result in subtle changes in the plant communities.

The SE $1 / 4$ of S28 is the richest and most diverse area overall within the PNA. It contains the bestdeveloped paloverde-saguaro-ironwood desertscrub with an understory of triangleleaf bursage. Ocotillo (Fouquieria splendens), pencil cholla (Opuntia 


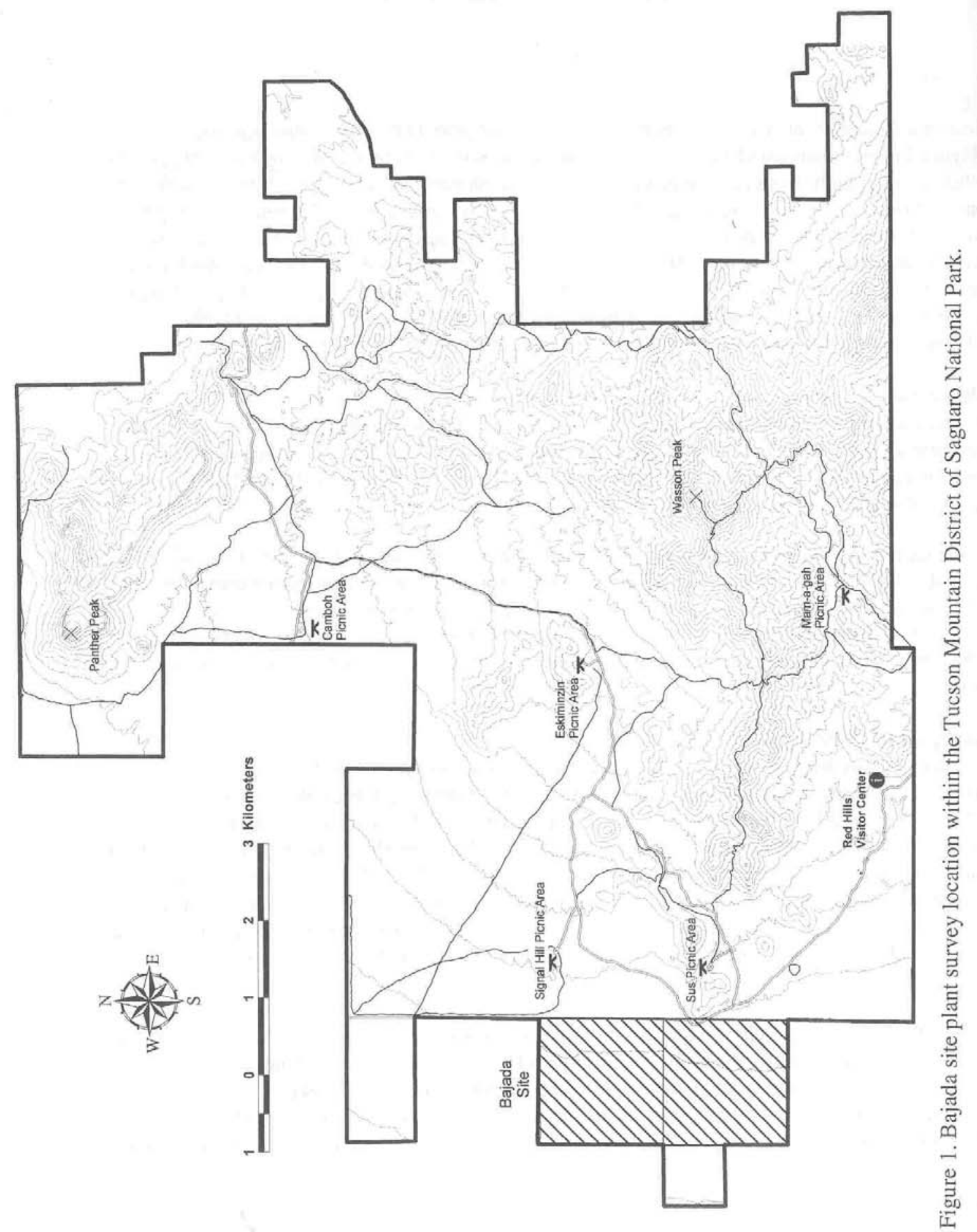


Moving from east to west in both sections, plant diversity decreases. Along the western boundary creosotebush dominates; saguaro (Carnegia gigantea), foothills paloverde (Parkinsonia microphylla), ironwood (Olneya tesota) and the other cacti occur occasionally. The vegetation is more open with trees and shrubs more widely spaced.

The northern portion of S21 has the least diversity of vegetation within the PNA. It contains primarily creosotebush intermixed with triangleleaf bursage. Fewer species of spring annuals were also found in this area, and several of these are characteristic of the Avra Valley, rather than the Tucson Mountains (e.g. Lupinus concinnus, Astragalus didymocarpus, Lotus humistratus, Cryptantha micrantha). White bursage (Ambrosia dumosa) was found only in this area. It is always restricted to fine-grained, often sandy soils in the vicinity of Tucson.

Four major east-west washes and a network of smaller tributaries crisscross the area. The larger drainages are lined with ironwood, foothills paloverde, and velvet mesquite (Prosopis velutina), sometimes with an understory of desert hackberry (Celtis pallida) and wolfberry (Lycium). In the spring dense stands of annual and perennial herbs were observed along the banks in the shade of trees and shrubs. In summer, although the herbs were concentrated along riparian corridors, there was less density and diversity. In many places perennial grasses, especially bush muhly (Muhlenbergia porteri), were common along the banks. These corridors exemplify the potential abundance of bush muhly in the absence of cattle grazing.

A different vegetation type occurs in the disturbed areas immediately adjacent to Ft. Lowell Road on the south, Sandario Road on the east, Manville Road on the north, and, to a lesser extent, the west boundary fence adjoining private property. A significantly greater number of exotic and weedy native species, mostly herbs, were found in these areas. Filaree (Erodium cicutarium), wild oats (Avena fatua), fescue grass (Bromus catharticus), wild barley (Hordeum murinum), littleseed canary grass (Phalaris minor), little mallow (Malva parviflora), and puncture vine (Tribulus terristris) were virtually restricted to roadsides.

\section{Introduced Species}

Fifteen introduced species were found in the PNA - twelve in spring and three in the summer. Only one, Mediterranean grass (Schismus barbatus), was abundant and widespread. This opportunistic annual was the most common species found in the spring throughout the study area due to an exceptional winter rainy season. All of the other introduced species were rare to locally common and most were restricted to roadsides or washes, microsites with extra available moisture. Most are spring annuals which will be more prevalent in wetter years like 1992. The only perennial and invasive species identified were buffel grass (Pennisetum ciliare) and Bermuda grass (Cynodon dactylon). Half a dozen clumps of buffel grass were observed, one near the west boundary fence, the others in or near the washes. A few localized patches of Bermuda grass were found on the roadcut above Sandario Road. 


\section{Addition to the Tucson Mountain Flora}

Yellow saucers (Malacothrix fendleri) was added to the Tucson Mountain flora (Rondeau 1991) as a result of the spring survey. A single plant of this native annual was observed along Sandario Road.

\section{Sensitive Species}

During plant surveys, care was taken to search for three species considered rare at the time: Thornber pincushion cactus (Mammillaria thornberi), Tumamoc globeberry (Tumamoca macdougalii), and night-blooming cereus (Peniocereus greggii). Since the time of the study, all three plants have lost their "sensitive" status.

Fifteen discrete populations of Thornber pincushion cactus (Mammillaria thornberi) were noticed and recorded in the spring of 1992. Eight populations encountered in summer may not all be different, since those found in spring were not marked. Therefore, the total number of populations observed is uncertain. The majority of plants was located along the boundary fences or roads in relatively disturbed habitat. Plants were found under both trees (ironwood and paloverde) and shrubs (creosotebush and triangleleaf bursage). Clumps consisted of from about five to fifty-five individuals. Most plants appeared healthy. One population, near Sandario Road, had many plants with brown tops and some were obviously dead. It is interesting that Thornbur pincushion cactus seems to favor disturbed sites within the PNA.

Tumamoc globeberry (Tumamoca macdougalii) and night-blooming cereus (Peniocereus greggii) were also sought during the summer survey. The globeberry was formerly federally listed as endangered, but intensive surveys funded after the listing turned up abundant plants. Thus the globeberry was delisted. The night-blooming cereus also appears to be more common than originally thought. Neither species was observed, but they are notoriously difficult to see in the field. Both species occur in nearby, similar habitats in the Tucson Mountains and may turn up with more intensive field work.

\section{Management Recommendations}

The two sections of Saguaro National Park west of Sandario Road in the Tucson Mountains support typical Arizona Upland Sonoran desertscrub. Few signs of human use were observed within the area, and the habitat is relatively undisturbed. No one was encountered away from the roads during the surveys. Several small piles of rusty trash were seen but appeared to be old dump sites. Dogs can and do crawl under the west boundary fence where private property abuts the park. While the perimeter of such a small area surrounded by roads and houses is bound to be disturbed, there is little evidence that the weedy and exotic species characteristic of these habitats are seriously invading the PNA.

On the other hand, Mediterranean grass, a spring annual which can readily inhabit the more arid flats, is abundant and widespread throughout the area on a seasonal basis. Control is unlikely; it will have to be tolerated. The species does not seem to be adversely affecting the growth of native spring annuals which were quite common the year of the survey. It is not likely, either, to replace six-weeks fescue (Vulpia octoflora), the most similar native grass, which prefers rockier substrates. 
One exotic species which does potentially pose a threat to the area is buffel grass. It is quite invasive and needs to be controlled before it gets a real foothold. Extensive areas in Sonora, Mexico have been intentionally converted from Sonoran Desert to tropical savanna by cultivation of fire-resistant buffel grass for livestock forage. Subsequent fires have decimated large areas of adjacent desertscrub. Buffel grass invasions are well underway in paloverdesaguaro desertscrub on Tumamoc Hill in the Tucson Mountains (T.L. Burgess personal communication, 1992). At this time it is uncommon in the PNA and could be eradicated by pulling out all plants and monitoring for new ones each year.

Although the PNA west of Sandario Road is an interesting and diverse area floristically, it is not very different from other low bajada areas within the Tucson Mountain Unit of Saguaro National Park. Thornbur pincushion cactus is thriving; Tumamoc globeberry and night-blooming cereus may be present. However, all of these sensitive species have been found to be more common and widespread than first believed. In addition, the area is rarely visited since no trails access the 520 ha $\left(2 \mathrm{mi}^{2}\right)$ and there are no obvious signs of human impact. The area does not appear to need additional protection beyond that already provided by being included within the park. 
Table 1. Bajada Site Checklist of Vascular Plants. Bajada is a Protected Natural Area within the Tucson Mountain District of Saguaro National Park. This list was compiled in 1992 by Rebecca Van Devender. Plant names follow Kartesz (1994).

$+=$ New addition to the Rondeau (1991) flora of the Tucson Mountains

$*=$ Introduced species

\{\}$=$ Observations made during 1992 surveys

\section{Acanthaceae}

Carlowrightia arizonica Gray

\section{Amaranthaceae}

Amaranthus fimbriatus (Torrey) Bentham ex Watson $\quad$ \{abundant, summer\}

Amaranthus palmeri Watson

Tidestromia lanuginosa (Nuttall) Standley

\section{Apiaceae}

Bowlesia incana Ruiz \& Pavón

Daucus pusillus Michaux

Spermolepis echinata (Nuttal ex DC.) Heller

\section{Aristolochiaceae}

Aristolochia watsonii Wooton \& Standley $\quad$ rare, wash, S21\}

\section{Asclepiadaceae}

Sarcostemma cynanchoides Decaisne ssp. hartwegii (Vail) R. Holm

\section{Asteraceae}

Acourtia nana (Gray) Reveal \& King $\quad$ rare, W fence

Acourtia wrightii (Gray) Reveal \& King \{rare, wash\}

Adenophyllum porphylloides (Strother) Gray

Ambrosia ambrosioides (Cavanilles) Payne

Ambrosia confertiflora DC.

Ambrosia deltoidea (Torrey) Payne \{abundant\}

Ambrosia dumosa (Gray) Payne $\quad\left\{\mathrm{N}^{1} 12, \mathrm{~S} 21\right\}$

Antheropeas lanosum Gray \& Rydberg \{abundant, spring, especially S28\}

Baccharis sarothroides Gray \{wash \& road\}

Baileya multiradiata Harvey \& Gray ex Gray $\quad$ rare, wash \& road\}

Brickellia coulteri Gray \{washes\}

Calycoseris wrightii Gray

Chaenactis stevioides Hooker \& Arnott

Encelia farinosa Gray ex Torrey

Erigeron divergens Torrey \& Gray

Filago arizonica Gray 
Filago californica Nuttall \{abundant, spring, especially S28\}

Filago depressa Gray

Heterotheca subaxillaris (Lam.) Britton and Rusby

Hymenoclea salsola Torrey \& Gray var. pentalepis (Rydberg) Benson

Hymenothrix wislizeni Gray \{roads, summer\}

Machaeranthera pinnatifida (Hooker) Shinners ssp. pinnatifida var. pinnatifida

+ Malacothrix fendleri Gray \{rare, spring, roadside\}

Microseris lindleyi (DC.) Gray

Monoptilon bellioides (Gray) H. M. Hall \{rare, spring W1/2, S21\}

Parthenium incanum (Kunth) \{wash\}

Pectis papposa Harv. \& Gray

Porophyllum gracile Bentham

Psilostrophe cooperi (Gray) Greene \{rare, wash,

Rafinesquia neomexicana Gray

Senecio flaccidus var monoensis (Greene) B.L. Turner \& T.M. Bark1.

*Sonchus oleraceus L. \{uncommon\}

Stephanomeria pauciflora (Torrey) A. Nelson

Stylocline micropoides Gray \{abundant, spring\}

Trixis californica Kellogg

Zinnia acerosa (DC.) Gray $\quad$ rare, S28\}

\section{Boraginaceae}

Amsinckia intermedia Fischer \& Meyer \{abundant, spring\}

Amsinckia tessellata Gray

Cryptantha angustifolia (Torrey) Greene

Cryptantha barbigera (Gray) Greene \{abundant, spring\}

Cryptantha decipiens (Jones) Heller

Cryptantha micrantha (Torrey) Johnst. $\quad$ rare, spring, $\mathrm{N}^{1} \frac{1}{2}, \mathrm{~S} 21$ \}

Cryptantha pterocarya (Torrey) Greene

Pectocarya heterocarpa (Johnston)

Pectocarya platycarpa (Munz and Johnston) Johnston \{abundant, spring\}

Pectocarya recurvata Johnston \{abundant, spring\}

\section{Brassicaceae}

*Brassica tournefortii Gouan \{local, spring, mostly N1/2, S21\}

Caulanthus lasiophyllus (H. \& A.) Payson \{abundant, spring\}

Descurainia pinnata (Walt.) Britt.

Draba cuneifolia Nuttall ex Torrey \& Gray $\quad$ rare, spring, wash

Lepidium lasiocarpum Nuttall

Lepidium virginicum var. medium (Greene) C.L. Hitchcock

Lesquerella gordoni (Gray) Watson \{rare, spring\}

*Sisymbrium irio L. \{occasional, spring, especially in washes\}

*Sisymbrium orientale L. \{uncommon, spring, wash in S28\}

Streptanthus carinatus Wright ex Gray

Thysanocarpus curvipes Hooker 


\section{Cactaceae}

Carnegia gigantea (Engelmann) Britton \& Rose $\quad$ especially E1/2, S28 \& SE $1 / 4$ S 21 \}

Echinocereus fendleri (Engelmann) F. Seitz var. fasciculatus (Engelmann ex B.D. Jackson) N.P. Taylor

Ferocactus wislizenii (Engelmann) Britton \& Rose

Mammillaria grahamii Engelmann var. grahamii [incl. M. microcarpa Engelmann] \{locally common SE $1 / 4, \mathrm{~S} 28\}$

Mammillaria thornberi Orcutt $\quad$ scattered under trees \& bushes, especially along park boundary\}

Opuntia acanthocarpa Engelmann \& Bigelow var. major (Engelmann \& Bigelow) L. Benson

Opuntia arbuscula Engelmann $\quad$ especially SE 1/4, S28\}

Opuntia fulgida Engelmann var. fulgida \{abundant, especially W1/2, S21 \& S28 but not far NW corner S21\}

Opuntia fulgida Engelmann var. mamillata (Schott ex Engelmann) Coulter

Opuntia x kelvinensis Grant \& Grant [= O. fulgida Engelmann x O. spinosior (Engelmann)

Toumey]

Opuntia leptocaulis DC. $\quad\{$ especially SE $1 / 4, \mathrm{~S} 28$ \}

Opuntia phaeacantha Engelmann

Opuntia spinosior (Engelmann) Toumey $\quad$ mostly S21\}

\section{Campanulaceae}

Nemacladus glanduliferus Jepson $\quad$ rare, spring, S28

\section{Caryophyllaceae}

Loeflingia squarrosa Nuttall $\quad$ rare, spring, S28\}

Silene antirrhina L. $\quad$ wwashes \& road, spring\}

\section{Chenopodiaceae}

Chenopodium neomexicanum Standley $\quad$ rare, summer\}

\section{Convolvulaceae}

Evolvulus alsinoides (L.) L. $\quad$ rare, summer\}

\section{Crassulaceae}

Crassula connata var. connato (Ruiz \& Pavón) Berger $\quad$ rare, spring, S28\}

\section{Cucurbitaceae}

Cucurbita digitata Gray $\quad$ \{local, summer, along west fence S28\}

\section{Euphorbiaceae}

Chamaesyce abramsiana (L.C. Wheeler) Koutnik \{abundant, summer, flats

Chamaesyce florida (Engelmann) Millspaugh

Chamaesyce gracillima (Watson) Millspaugh

Chamaesyce hyssopifolia (L.) Small

Chamaesyce micromera (Boiss). Wooton \& Standley 
Chamaesyce polycarpa (Bentham) Millspaugh ex Parish

Chamaesyce setiloba (Engelmann ex Torrey) Millspaugh ex Parish

Jatropha cardiophylla (Torrey) Mueller-Argoviensis

\section{Fabaceae}

Acacia constricta Bentham

Acacia greggii Gray

Astragalus didymocarpus Hooker \& Arnott $\quad\{\mathrm{N} 1 / 2, \mathrm{~S} 21\}$

Astragalus lentiginosus Douglas ex Hooker var. australis Barneby

Calliandra eriophylla Bentham

Lotus humistratus Greene $\quad\left\{\mathrm{N} 1 \frac{2}{2}, \mathrm{~S} 21\right\}$

Lotus strigosus (Nuttall) Greene var. tomentellus (Greene) Isely

Lupinus concinnus Agardh. $\quad$ especially N1/2, S21\}

Lupinus sparsiflorus Bentham

Marina parryi (Torrey \& Gray ) Barneby

Nissolia schottii (Torrey) Gray $\quad$ rare, wash

Olneya tesota Gray

Parkinsonia microphylla Torrey $\quad$ especially E $1 / 2, \mathrm{~S} 28 \&$ SE $1 / 4, \mathrm{~S} 21\}$ \}

Prosopis velutina Wooton

Senna covesii (Gray) Irwin \& Barneby

\section{Fouquieriaceae}

Fouquieria splendens Engelmann $\quad$ drops out on W side \& N side

\section{Geraniaceae}

*Erodium cicutarium (L.) L'Her. ex Ait. \{mostly in disturbed areas, spring, along roads \& boundary fences

Erodium texanum Gray

\section{Hydrophyllaceae}

Eucrypta micrantha (Torrey) Heller

Nama hispidum Gray $\quad$ rare, spring, along $\mathrm{N}$ fence, S21\}

Phacelia distans Bentham $\quad$ abundant, spring, washes \& under trees\}

\section{Krameriaceae}

Krameria grayi Rose \& Painter

\section{Lamiaceae}

Hyptis emoryi Torrey

Salvia columbariae Bentham

\section{Loasaceae}

Mentzelia affinis Greene 


\section{Malpighiaceae}

Janusia gracilis A. Gray

Malvaceae

Abutilon incanum (Link) Sweet

Abutilon malacum Watson

Herissantia crispa (L.) Brizicky

*Malva parviflora L. $\quad$ \{local, spring, along road\}

Sida abutifolia Miller

Sphaeralcea coulteri (Watson) Gray

Sphaeralcea laxa Wooton \& Standley

\section{Molluginaceae}

Mollugo cerviana (L.) Seringe $\quad$ rare, summer\}

\section{Nyctaginaceae}

Allionia incarnata L.

Boerhavia coccinea Miller \{rare, summer, near road

Boerhavia coulteri (Hooker f.) Watson

Boeraavia intermedia Jones \{abundant, summer\}

Boerhavia scandens (L.)

Mirabilis bigelovii Gray $\quad$ rare, wash, S21\}

\section{Oleaceae}

Menodora scabra Gray

\section{Onagraceae}

Camissonia chamaenerioides (Gray) Raven

\section{Oxalidaceae}

Oxalis albicans Kunth ssp. pilosa (Nuttall) Eiten

Oxalis alpina (Rose) Rose ex Knuth

\section{Papaveraceae}

Eschscholtzia californica ssp. mexicana (Greene) C. Clark

\section{Pedaliaceae}

Proboscidea altheaefolia (Bentham) Decne.

Proboscidea parviflora (Wooton) Wooton \& Standley $\quad$ Rare, summer, N fence, S21\}

\section{Plantaginaceae}

Plantago ovata Forsk

Plantago patagonica Jacq. 


\section{Poaceae}

Aristida adscencionis L.

Aristida purpurea Nuttal

Aristida ternipes Cavanilles

*Avena fatua L. \{locally common along road, spring\}

Bouteloua aristidoides (Kunth) Grisebach \{abundant, summer, flats\}

Bouteloua barbata Lagasca

Bouteloua curtipendula (Michaux) Torrey \{rare, summer, wash\}

Bouteloua repens (Kunth) Scribner \& Merrill

Boutelous rothrockii Vasey \{rare, summer, wash\}

Brachiaria arizonica (Scribner \& Merrill) S.T. Blake

Bromus carinatus Hooker \& Arnott

*Bromus catharticus Vahl. \{rare along road, spring\}

*Bromus madritensis L. \{occasional to uncommon, spring\}

* Cynodon dactylon (L.) Persoon $\quad$ rare, roadside\}

Digitaria californica (Bentham) Henrard

*Eragrostis cilianensis (All.) Janchen ex Lut. \{rare, summer, wash\}

Erioneuron pulchellum (Kunth) Tateoka

Heteropogon contortus (L.) Beauvois ex Roemer \& Schultes \{rare, summer, wash

Hordeum murinum L. ssp. glaucum (Steud.) Tzvelev \{locally along road, spring\}

Leptochloa mucronata (Michaux) Kunth

Muhlenbergia microsperma (DC.) Trin.

Muhlenbergia porteri Scribner \{abundant, washes\}

Muhlenbergia rigens (Bentham) Hitchcock

Panicum hirticaule Presl. $\quad$ rare, summer, wash\}

*Pennisetum ciliare (L.) Link \{occasional, west fence \& washes\}

*Phalaris minor Retz. $\quad$ \{rare, spring, along road\}

Poa bigelovii Vasey \& Scribner

*Schismus barbatus (Loefl. ex L.) Thell. $\quad$ \{abundant, spring, far N end, S21 where dominant with creosotebush\}

Setaria leucopila (Scribner \& Merr.) K. Schum.

Sporobolis cryptandrus (Torrey) Gray \{rare, summer, wash\}

Vulpia octoflora (Walt.) Rydberg \{abundant, spring, especially S28\}

\section{Polygonaceae}

Chorizanthe brevicornu Torrey $\quad$ mostly N1/2, S21\}

Eriogonum abertianum Torrey \{rare, summer, wash\}

Eriogonum deflexum Torrey

\section{Ranunculaceae}

Delphinium scaposum Greene

\section{Rhamnaceae}

Ziziphus obtusifolia (Hooker ex Torrey \& Gray) Gray var. canescens (Gray) M. C. Johnston \{rare, S28\} 


\section{Scrophulariaceae}

Nuttallanthus texanus (Scheele) D.A. Sutton

Castilleja exerta ssp. exerta

Penstemon parryi (Gray) Gray \{washes\}

\section{Solanaceae}

Datura discolor Bernh.

Datura wrightii Regel

Lycium berlandieri Dunal

Lycium sp. $\quad$ \{washes (vegetative only)\}

Nicotiana trigonophylla Dunal

Physalis crassifolia Bentham

\section{Sterculiaceae}

Ayenia filiformis Watson

\section{Ulmaceae}

Celtis pallida Torrey

Urticaceae

Parietaria hespera Hinton

\section{Viscaceae}

Phoradendron californicum Nuttall

\section{Zygophyllaceae}

Kallstroemia californica (Wats.) Vail

Larrea Cav. tridentata (Sessé \& Moc. ex DC.) Coville var. tridentata

*Tribulus terrestris L. $\quad$ occasional, summer, roadside\} 


\title{
Box Canyon (Rincon Mountain District)
}

\author{
By Mark Fishbein, Victor Steinman, and Amy Johnson
}

The initial survey of Box Canyon, within the Rincon Mountain District of Saguaro National Park, was conducted September 3-5, 1993. We made follow-up observations of spring annuals April 13-14, 1994. Data were collected by walking transects. The goals of the study were to comment on the status of introduced species in the area, note species new to the Bowers and McLaughlin (1987) flora of the Rincon Mountains, and develop the checklist.

The study area (Figure 2) is approximately $8 \mathrm{~km}$ long from the mouth of the wash (T15S, R16E, $\mathrm{NE} 1 / 4$ of Section 8) to the extreme box-canyon wall one-half mile below Juniper basin (T14S, R17E, SW $1 / 4$ of Section 30). The canyon descends approximately $925 \mathrm{~m}$ from Juniper Basin at $1825 \mathrm{~m}$ to the mouth of the wash at $900 \mathrm{~m}$ elevation.

Box Canyon was designated as a Protected Natural Area in the park's General Management Plan (NPS 1987) because it contains perennial pools and rich riparian habitat. Access to this steep canyon is limited, and the boundary fence crossing the lower wash has successfully discouraged entrance by horses and cattle. No trails reach Box Canyon, but motivated hikers can get to it via the Tanque Verde Ridge trail that parallels the entire canyon just above its northern edge (Figure 2).

\section{Vegetation}

Due to its elevational gain and year-round water, the canyon supports a variety of vegetation communities: Saguaro-Foothills Palo Verde Desertscrub, Desert Riparian, Desert-grassland, and Oak Woodland. The Wildhorse Canyon chapter in this report contains detailed descriptions (see page 35) of these associations. The Box Canyon survey identified 387 species from 79 families. Survey results are listed in Table 2 at the end of this chapter. We deposited one set of voucher specimens in the University of Arizona herbarium and a second set of the plants new to the Saguaro National Park checklist in the park herbarium.

\section{Introduced Species}

Twenty-four introduced species were found within the Box Canyon PNA, among them, 15 grasses. The annual red brome (Bromus madritensis) and the perennial Bermuda grass (Cynodon dactylon) were particularly widespread throughout the canyon. Wild oats (Avena fatua) was common within the lower wash, and other grasses such as barley (Critesion murinum) and Lehmann lovegrass (Eragrostis lehmanniana) occurred throughout the streambed. The big, bunchy perennials buffel grass (Pennisetum ciliare) and fountain grass (P. setaceum) are also present.

Annuals such as camphorweed (Centaurea melitensis) and sow thistles (Sonchus asper and S. oleraceus) had patchy distributions but were present throughout the canyon. These composites are very opportunistic and can grow in a variety of areas. 


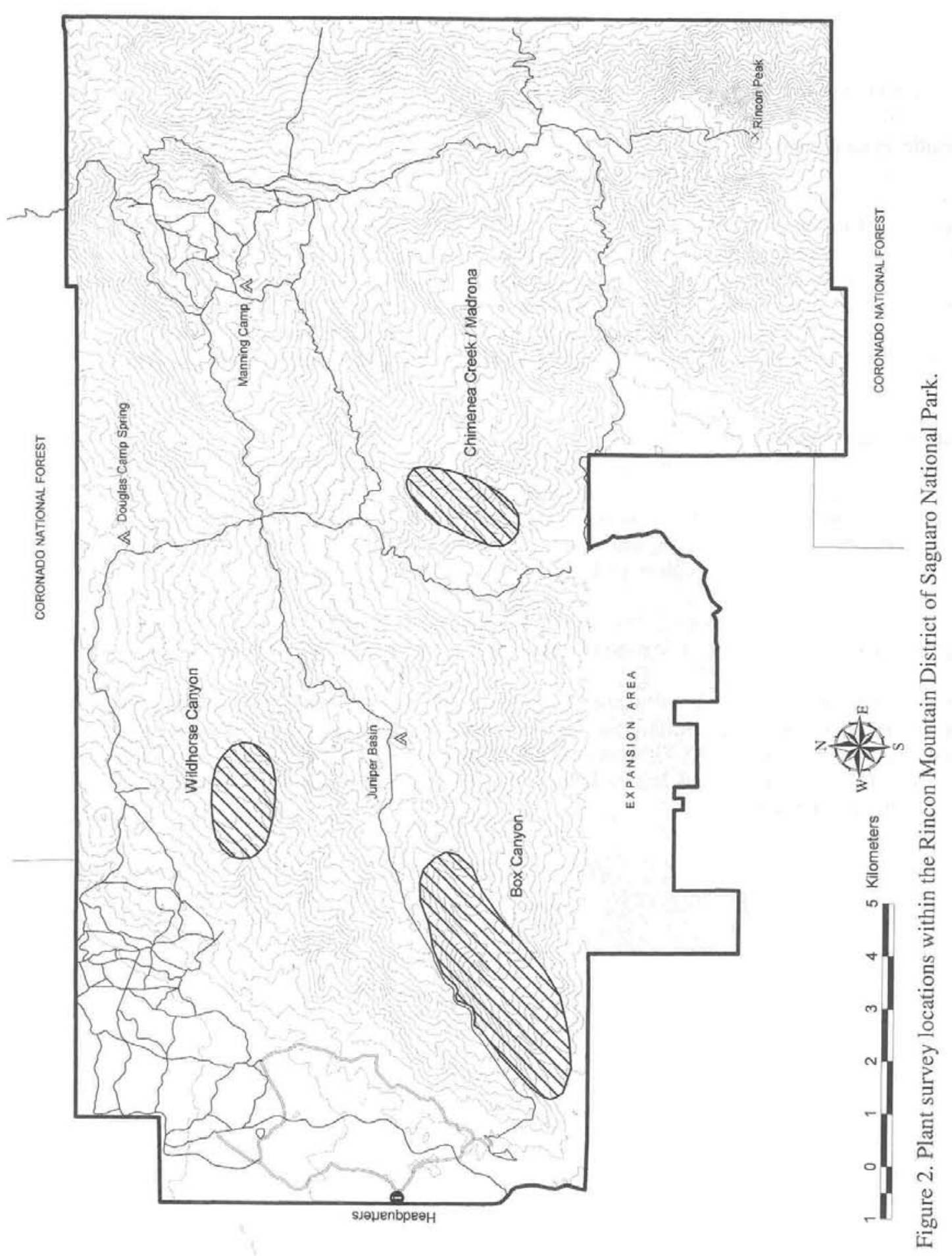


Other exotic species encountered were uncommon to rare; at this time they are not considered to be threatening or damaging to the ecosystems they inhabit.

\section{Additions to the Flora}

Nine species new to the Bowers and McLaughlin (1987) Rincon Mountains flora have been marked with a plus sign $(+)$ on the plant list. Seven of these species are natives: Ruellia nudiflora, Mirabilis oxybaphoides, Pectocarya setosa, Bulbostylis cf. funckii, Cyperus cf. parishii, Bouteloua eludens, and Panicum lepidulum. The two sedges (family Cyperaceae), Bulbosylis funckii and Cyperus parishii, were found in moist seepages within the desert riparian zone. The often invasive buffel grass (Pennisetum ciliare) was one of two introduced species new to the flora. The other was the prickly pear known as Indian fig (Opuntia ficus-indica), found in the lower canyon Desertscrub, where we saw one particularly large, well-established specimen.

\section{Management Suggestions}

The results of this study support the Protected Natural Area designation for Box Canyon. Over its elevational extent, the canyon is home to about 40\% (387/995) of all the plant species known from the Rincon Mountains. Perennial water maintains a rich riparian flora and makes the canyon important terrestrial and aquatic wildlife habitat. Continuing the lack of formal access will help preserve this valuable Saguaro National Park drainage.

There is a thriving feather bush (Lysiloma watsoni) population in Box Canyon. Monitoring this population is a priority, given it is at the northern edge of the species' distribution.

Finally, we suggest that measures be taken to eradicate buffel grass (Pennisetum ciliare) and fountain grass (P. setaceum). Buffel grass has been noted for the first time in this survey, and fountain grass was new in 1987 (Bowers and McLaughlin 1987). Though steep canyon walls serve as natural barriers to plant dispersal, these invaders will likely migrate up the canyon with time, given no intervention. 
Table 2. Box Canyon Checklist of Vascular Plants. Box Canyon is a Protected Natural Area within the Rincon Mountain District of Saguaro National Park. This list was compiled in 1993 and 1994 by Mark Fishbein, Victor Steinman, and Amy Johnson. Plant names follow Kartesz (1994).

$+=$ New addition to the flora of the Rincon Mountains of Bowers and McLaughlin (1987)

$*=$ Introduced species

\section{Acanthaceae}

Anisacanthus thurberi (Torrey) Gray

Carlowrightia arizonica Gray

+ Ruellia nudiflora (Englelmann \& Gray)

Urban

Siphonoglossa longiflora (Torrey) Gray

\section{Adiantaceae}

Adiantum capillis-veneris L.

Astrolepis cochisensis (Goodding)

Bentham \& Windham

Astrolepis sinuata (Lagasca) Bentham \&

Windham

Bommeria hispida (Metternich ex Kuhn)

Underwood

Cheilanthes bonariensis (Willdenow) Proctor

Cheilanthes fendleri Hooker

Cheilanthes lindheimeri Hooker

Cheilanthes wootonii Maxon

Cheilanthes wrightii Hooker

Notholaena lemmonii D.C. Eaton

Notholaena standleyi Maxon

Pellaea truncata Goodding

Pellaea wrightiana Hooker

Pentagramma triangularis (Kaulfuss)

Yatskievych, Windham \& Wollenweber

ssp. maxonii (Weatherby)

Yatskievych, Windham \& Wollenweber

\section{Agavaceae}

Agave cf. palmeri Engelmann

Agave schottii Engelmann var. schottii

Dasylirion wheeleri S. Watson

Nolina microcarpa S. Watson

Yucca schottii Engelmann

\section{Amaranthaceae}

Amaranthus palmeri S. Watson

Gomphrena sonorae Torrey

\section{Amaryllidaceae}

Zephyranthes longifolia Hemsley

Anacardiaceae

Rhus trilobata (Nuttall)

var. pilosissima Engelmann

Apocynaceae

Haplophyton cimicidum DC.

\section{Araliaceae}

Aralia humilis Cavanilles

\section{Aristolochiaceae}

Aristolochia watsonii Wooton \& Standley

Asclepiadaceae

Asclepias linaria Cavanilles

Asclepias nyctaginifolia Gray

Matelea arizonica (Gray) Shinners

Matelea parviflora (Torrey) Woodson

Sarcostemma cynanchoides Decaisne

ssp. hartwegii (Vail) R. Holm

\section{Asteraceae}

Acourtia thurberi (Gray) Reveal \& King Acourtia wrightii (Gray) Reveal \& King Ambrosia ambrosioides (Cavanilles) Payne Ambrosia confertiflora DC.

Antheropeas lanosum (Gray) Rydberg

Artemisia ludoviciana Nuttall

ssp. albula (Wooton) Keck

Baccharis brachyphylla Gray 
Baccharis salicifolia (Ruiz \& Pavón) Persoon

Baccharis sarothroides Gray

Baccharis thesioides Kunth

Bahia absinthifolia Bentham

var. dealbata (Gray) Gray

Baileya multiradiata Harvey \& Gray ex Gray

Bebbia juncea (Bentham) Greene

Brickellia betonicifolia Gray

Brickellia californica (Torrey \& Gray) Gray

Brickellia coulteri Gray

Brickellia venosa (Wooton \& Standley) B.L.

Robinson

Carminatia tenuiflora DC.

Carphochaete bigelovii Gray

+ Centaurea melitensis L.

Cirsium neomexicanum Gray

Conyza canadensis (L.) Cronquist

Coreocarpus arizonicus (Gray) Blake

Cosmos parviflorus (Jacquin) Persoon

Encelia farinosa Gray

Ericameria laricifolia (Gray) Shinners

Erigeron divergens Torrey \& Gray

Erigeron oreophilus Greenman

Eupatorium solidaginifolium Gray

Gnaphalium leucocephalum Gray

Gnaphalium canescens DC.

Guardiola platyphylla Gray

Gymnosperma glutinosum (Sprengel)

Lessing

Heliomeris longifolia var. annua (Jones)

Yates

Heterotheca subaxillaris (Lamarck) Britton \& Rusby

Hymenoclea monogyra Torrey \& Gray ex Gray

Hymenothrix wrightii Gray

Isocoma tenuisecta Greene

Lasthenia californica DC. ex Lindley

Machaeranthera canescens var. incana (Lindl.) Gray

Machaeranthera gracilis (Nuttall) Shinners

Machaeranthera pinnatifida (Hooker)

Shinners ssp. pinnatifida var. pinnatifida

Machaeranthera tagetina Greene

Microseris lindleyi (DC.) Gray
Parthenise mollis Gray

Parthenium incanum Kunth.

Perityle lemmoni (Gray) Macbride

Porophyllum gracile Bentham

Psilostrophe cooperi (Gray) Greene

Senecio lemmoni Gray

*Sonchus asper (L.) Hill

*Sonchus oleraceus L.

Stephanomeria pauciflora (Torrey) A.

Nelson

Tagetes lemmonii Gray

Tagetes micrantha Cavanilles

Thymophylla pentachaeta var. pentachaeta

Trixis californica Kellogg

Viguiera dentata (Cavanilles) Sprengel

var. lancifolia Blake

Zinnia acerosa (DC.) Gray

\section{Boraginaceae}

Amsinckia intermedia Fisch. \& Mey.

Cryptantha angustifolia (Torrey) Greene

Cryptantha barbigera (Gray) Greene

Cryptantha micrantha (Torrey) I.M. Johnston

Cryptantha pterocarya (Torrey) Greene

var. cycloptera (Greene) Macbride

Lappula occidentalis (Watson) Greene

Pectocarya platycarpa (Munz \& I. M.

Johnston)

Munz \& I. M. Johnston

Pectocarya recurvata I. M. Johnston

Pectocarya setosa Gray

\section{Brassicaceae}

Arabis perennans S. Watson

Caulanthus lasiosphyllus (Hooker \& Arnott)

Payson

var. lasiophyllus

Lepidium virginicum $\mathrm{L}$.

var. medium (Greene) C. L. Hitchcock

*Sisymbrium irio L.

Thysanocarpus curvipes Hooker

Cactaceae

Carnegia gigantea (Engelmann) Britton \& Rose 
Echinocereus fendleri Engelmann var. fasciculatus

Echinocereus fendleri (Engelmann)

Engelmann var. fendleri

Escobaria vivpara var. bisbeeana (Orcutt) D.R. Hunt

Ferocactus wislizenii (Engelmann) Britton \& Rose

Mammillaria grahamii Engelmann var. oliviae (Orcutt) Benson

Mammillaria heyderi Muhl. var. macdougalii (Rose) L. Benson

Opuntia arbuscula Engelmann

Opuntia bigelovii Engelmann

Opuntia chlorotica Engelmann \& Bigelow

Opuntia engelmannii Salm-Dyck

Opuntia engelmannii var. engelmannii SalmDyck

*+Opuntia ficus-indica (L.) Mill.

Opuntia fulgida Engelmann var. fulgida

Opuntia fulgida Engelmann

var. mammillata (Schott) Coulter

Opuntia leptocaulis DC.

Opuntia phaeacantha Engelmann

var. major (Engelmann)

Opuntia spinosior (Engelmann) Toumey

Opuntia versicolor Engelmann ex Coulter

\section{Campanulaceae}

Triodanis perfoliata (L.) Nieuwland var. biflora (Ruiz \& Pavón) Bradley

Triodanis perfoliata (L.) Nieuwland var. perfoliata

\section{Caryophyllaceae}

*Cerastium gracile Dufour

Cerastium texanum Britton

Silene antirrhina $\mathrm{L}$.

\section{Chenopodiaceae}

Atriplex canescens (Pursh) Nuttall

\section{Commelinaceae}

Commelina erecta $\mathrm{L}$.

Tradescantia occidentalis (Britton) Smythe

\section{Convolvulaceae}

Evolvulus alsinoides (L.) L.

Evolvulus arizonicus Gray

Ipomoea barbatisepala Gray

Ipomoea costellata Torrey

Jacquemontia pringlei Gray

Crassulaceae

Sedum cockerellii Britton

Crossosomataceae

Crossosoma bigelovii S. Watson

\section{Cucurbitaceae}

Cucurbita digitata Gray

Echinopepon wrightii (Gray) S. Watson

Marah gilensis Greene

\section{Cupressaceae}

Juniperus deppeana Steudel

\section{Cyperaceae}

+Bulbostylus cf. funckii (Steudel) C. D.

Clarke

Carex chihuahuensis Mackenzie

* Cyperus esculentus L.

+Cyperus cf. parishii Britton

Cyperus pringlei Britton

Cyperus squarrosus L.

Cyperus wrightii Britton

\section{Dryopteridaceae}

Woodsia cochisensis Windham

\section{Ephedraceae}

Ephedra trifurca Torrey ex Watson

\section{Ericaceae}

Arctostaphylos pungens Kunth 
Euphorbiaceae

Acalypha neomexicana Mueller-Argoviensis

Argythamnia neomexicana Mueller-

Argoviensis

Chamaesyce florida (Engelmann) Millspaugh

Chamaesyce hyssopifolia (L.) Standley

Chamaesyce melanadenia (Torrey)

Millspaugh

Euphorbia heterophylla L.

Jatropha cardiophylla (Torrey) Mueller-

Argoviensis

Tragia nepetifolia Cavanilles

\section{Fabaceae}

Acacia angustissima (Millspaugh) Kuntze

Acacia constricta Bentham

Acacia greggii Gray

Amorpha fruticosa $\mathrm{L}$.

Astragalus nothoxys Gray

Calliandra eriophylla Bentham

Chamaecrista nictitans (L.) Moench

var. leptadenia Gandhi \& Hatch

Cologania angustifolia Kunth

Coursetia glandulosa Gray

Crotalaria pumila Ortega

Crotolaria sagittalis L.

Dalea albiflora Gray

Dalea filiformis Gray

Dalea pringlei Gray

Dalea pulchra Gentry

Desmodium batocaulon Gray

Desmodium rosei Schubert

Galactia wrightii Gray

Lathyrus graminifolius (S. Watson) White

Lotus humistratus Greene

Lotus plebeius (Brand) Bradley

Lotus rigidus (Bentham) Greene

Lupinus concinnus Agardh.

ssp. orcuttii (S. Watson) Dunn

Lysiloma watsoni Rose

Macroptilium gibbosifolium (Ortega) A.

Delgado

Mimosa aculeaticarpa Ortega

var. biuncifera (Bentham) Barneby
Nissolia schottii (Torrey) Gray

Parkinsonia microphylla Torrey

Phaseolus acutifolius Gray

Prosopis velutina Wooton

Tephrosia tenella Gray

Vicia americana Muhl. ex Willdenow ssp. americana

Vicia ludoviciana Nuttall

Fagaceae

Quercus arizonica Sargent

Quercus emoryi Torrey

Quercus oblongifolia Torrey

Quercus turbinella Greene

\section{Fouquieriaceae}

Fouquieria splendens Engelmann

Garryaceae

Garrya wrightii Torrey

\section{Gentianaceae}

Centaurium nudicaule (Engelmann) B. L.

Robinson

Hydrophyllaceae

Eucrypta chrysanthemifolia (Bentham)

Greene

var. bipinnatifida (Torrey) Constance

Nama hispidum Gray

Phacelia distans Bentham

Iridaceae

Sisyrinchium cernuum (Bicknell) Kearney

\section{Juglandaceae}

Juglans major (Torrey) Heller

\section{Juncaceae}

Juncus ensifolius Wiks.

Juncus tenuis Willdenow

Juncus sp. 


\section{Lamiaceae}

Hedeoma nana (Torrey) Briquet ssp. nana

Hyptis emoryi Torrey

Salvia columbariae Bentham

Stachys coccinea Jacquin

\section{Liliaceae}

Dichelostemma capitatum (Bentham) Wood

\section{Loasaceae}

Mentzelia albicaulis (Douglas ex Hooker)

Torrey \& Gray

\section{Lythraceae}

Cuphea wrightii Gray

\section{Malpighiaceae}

Janusia gracilis Gray

\section{Malvaceae}

Abutilon abutiloides (Jacquin) Garcke ex Britton \& Wilson

Abutilon incanum (Link.) Sweet

Abutilon mollicomum (Willdenow) Sweet

Abutilon reventum S. Watson

Anoda abutiloides Gray

Anoda cristata (L.) Schlechtendal

Gossypium thurberi Todaro

Herissantia crispa (L.) Brizicky

Hibiscus coulteri Harvey ex Gray

Rhynchosida physocalyx (Gray) Fryxell

Sphaeralcea fendleri Gray

Sphaeralcea laxa Wooton \& Standley

\section{Molluginaceae}

Mollugo verticillata $\mathrm{L}$.

\section{Moraceae}

Morus microphylla Buckley

Nyctaginaceae

Allionia incarnata L.

Boerhavia diffusa L.

Boerhavia scandens L.
Mirabilis oblongifolia (Gray) Heimerl.

+Mirabilis oxybaphoides (Gray) Gray

Oleaceae

Fraxinus velutina Torrey

Menodora scabra Gray

Onagraceae

Epilobium canum (Greene) Raven

ssp. latifolium (Hooker) Raven

Oenothera caespitosa Nuttall

Oxalidaceae

Oxalis albicans Kunth ssp. pilosa (Nuttall)

Eiten

\section{Papaveraceae}

Corydalis aurea Willdenow

Passifloraceae

Passiflora mexicana Jussieu

Phytolaccaceae

Rivina humilis L.

Pinaceae

Pinus discolor Bailey \& Hawksworth

Plantaginaceae

Plantago patagonica Jacquin

Plantago virginica $\mathrm{L}$.

\section{Platanaceae}

Platanus wrightii S. Watson

\section{Plumbaginaceae}

Plumbago scandens L.

\section{Poaceae}

Aristida adscencionis L.

Aristida ternipes var. hamulosa (Henr.) Trent

Aristida pupurea Nuttall

var. longeseta (Steudel) Vasey

Aristida ternipes Cavanilles

* Avena fatua L. 
Bothriochloa barbinodis (Lagasca) Herter

Bouteloua aristidoides (Kunth) Grisebach

Bouteloua barbata Lagasca

Bouteloua chondrosioides (Kunth)

Bentham ex Watson

Bouteloua curtipendula (Michaux) Torrey

+Bouteloua eludens Griffiths

Bouteloua hirsuta Lagasca

Bouteloua repens (Kunth) Scribner \& Merrill

Bouteloua rothrockii Vasey

Bromus carinatus Hooker \& Arnott

*Bromus madritensis L.

Chloris virgata Swartz.

* Cynodon dactylon (L.) Persoon

Digitaria californica Henrard

Elymus elymoides (Rafinesque) Swezey

Enneapogon desvauxii P. Beauvois

* Eragrostis cilianensis (Allard)

Vignolo-Lutati ex Janchen

Eragrostis intermedia Hitchcock

* Eragrostis lehmanniana Nees

Eragrostis pectinacea (Michaux) Nees ex

Steudel

var. pectinacea

Eragrostis pectinacea (Michaux) Nees var. miserrima (Fournier) J. Reeder

Eriochloa aristida Vasey

Eriochloa lemmonii Vasey \& Scribner

Erioneuron pulchellum (Kunth) Tateoka

Heteropogon contortus (L.) Beauvois ex Roemer \& Schultes

*Hordeum murinum ssp. leporium (Link)

Arcang.

Hordeum pusillum Nuttall ssp. pusillum

Leptochloa dubia (Kunth) Nees

Leptochloa fascicularis (Lamarck) Gray

Leptochloa filiformis (Lamarck) P. Beauvois

Lycurus setosus (Nuttall) C. Reeder

Muhlenbergia arizonica Scribner

Muhlenbergia dumosa Scribner

Muhlenbergia emersleyi Vasey

Muhlenbergia minutissima (Steudel) Swallen

Muhlenbergia pauciflora Buckley

Muhlenbergia porteri Scribner

Muhlenbergia rigens (Bentham) Hitchcock
Muhlenbergia sinuosa Swallen

Muhlenbergia tenuifolia (Kunth) Trin.

Panicum bulbosum Kunth

Panicum capillare L.

+ Panicum lepidum Hitchcock \& Chase

$+*$ Pennisetum ciliare (L.) Link

* Pennisetum setaceum (Forskal) Chiov.

Phalaris caroliniana Walt.

Piptochaetium fimbriatum (Kunth) Hitchcock

* Poa pratensis L.

*Polypogon monspeliensis (L.) Desfontaines

* Rhynchelytrum repens (Willdenow) C.E.

Hubbard

*Schismus barbarus (L.) Pearson

Schizachyrium cirratum (Hackel) Wooton \&

Standley

Schizachyrium sanguineum (Retzius) Alston

Setaria macrostachya Kunth

*Sorghum halapense (L.) Persoon

Sporobolus contractus A.S. Hitchcock

Sporobolus wrightii Munro ex Scribner

Stipa sp.

Vulpia microstachys (Nuttall) Bentham

var. pauciflora (Scribner) Lonard \&

Gould

Vulpia octoflora (Walter) Rydberg

var. hirtella (Piper) Henr.

Vulpia octoflora (Walter) Rydberg var.

octoflora

\section{Polemoniaceae}

Eriastrum diffusum (Gray) Mason

Ipomopsis multiflora (Nuttall) V. Grant

Linanthus aureus (Nuttall) Greene

Phlox tenuifolia E. Nelson

\section{Polygonaceae}

Eriogonum pharmaceoides Torrey

var. pharmaceoides

Eriogonum polycladon Bentham

Eriogonum thurberi Torrey

Eriogonum wrightii Torrey ex Bentham var.

wrightii

*Polygonum aviculare L.

* Rumex crispus L. 
Rumex hymenosepalus Torrey

\section{Portulacaceae}

Claytonia perfoliata Donn ex Willdenow Portulaca suffrutescens Engelmann

Talinum paniculatum (Jacquin) Gaertner

Primulaceae

Androsace occidentalis Pursch

Ranunculaceae

Anemone tuberosa Rydberg

Clematis ligusticifolia Nuttall

Delphinium scaposum Greene

\section{Rhamnaceae}

Condalia warnockii M.C. Johnston

Rhamnus crocea Nuttall

ssp. ilicifolia (Kellogg) C.B. Wolf

Ziziphus obtusifolia (Hooker ex Torrey \&

Gray) Gray

Rosaceae

Vauquelinia californica (Torrey) Sargent

\section{Rubiaceae}

Bouvardia ternifolia (Cavanilles)

Schlechtendal

* Galium aparine L.

Galium microphyllum Gray

Galium wrightii Gray

\section{Salicaceae}

Populus fremontii S. Watson

Salix exigua Nuttall

Salix gooddingii Ball

\section{Sapindaceae}

Dodonea viscosa (L.) Jacquin

Sapindus saponaria

var. drummondii L. (Hooker \& Arnott) L.

Benson

\section{Sapotaceae}

Sideroxylon lanuginosum Michaux

\section{Saxifragaceae}

Heuchera sanguinea Engelmann

Scrophulariaceae

Castilleja austromontana Standley \& Blumer

Castilleja tenuiflora Bentham

Maurandella antirrhiniflora

(Humboldt \& Bonpland ex Willdenow)

Rothm.

Mecardonia procumbens (P. Mill.) Small

Mimulus guttatus DC.

Nuttallanthus texanus (Scheele) D.A. Sutton

Penstemon barbatus (Cavanilles) Roth

Penstemon parryi (Gray) Gray

Stemodia durantifolia (L.) Swartz

Veronica peregrina L.

ssp. xalapensis (Kunth) Pennell

\section{Solanaceae}

Datura discolor Bernh.

Lycium berlandieri Dunal

Lycium exsertum Gray

Nicotiana trigonophylla Dunal

Physalis crassifolia Bentham

var. versicolor (Rydberg) Waterfall

Physalis cf. hederifolia Gray

Solanum douglasii Dunal

\section{Sterculiaceae}

Ayenia compacta Rose

Ayenia microphylla Gray

\section{Typhaceae}

Typha cf. domingensis Persoon

\section{Ulmaceae}

Celtis pallida Torrey

Celtis laevigata Willdenow

var. reticulata (Torrey) L. Benson

\section{Urticaceae}

Parietaria hespera Hinton 


\section{Valerianaceae \\ Plectritis ciliosa (Greene) Jepson ssp. insignis (Suksdorff) Morey \\ Verbenaceae \\ Aloysia wrightii A.A. Heller ex Abrams \\ Glandularia bipinnatifida (Nuttall) Nuttall var. bipinnatifida}

\section{Viscaceae}

Phoradendron californicum Nuttall
Phoradendron villosum ssp. coryae

(Trelease) Wiens

+Phoradendron juniperinum Engelmann

\section{Vitaceae}

Vitis arizonica Engelmann

Zygophyllaceae

Larrea tridentata (Sesse \& Mocino ex DC.) Coville 


\section{Chimenea and Madrona Canyons (Rincon Mountain District)}

Surveys of Chimenea Canyon, within the Rincon Mountain District of Saguaro National Park, were conducted during April, May, and October of 1994. The Madrona Canyon surveys were conducted in October 1995. Portions of the two canyons form a Protected Natural Area in the park on the west slope of the Rincon Mountains. As shown in Figure 2, the drainages run parallel to one another, heading southwest in the upper reaches then turning south at lower elevation. Elevation drops from approximately $1600 \mathrm{~m}$ to $1150 \mathrm{~m}(5200 \mathrm{ft}$ to $3800 \mathrm{ft})$ within the study areas.

The species lists for Chimenea and Madrona Canyons were compiled by walking selected transects and recording all species encountered. Workers collected voucher specimens for all species. A complete set of specimens was deposited in the University of Arizona herbarium. Vouchers for species new to Saguaro National Park were brought to the park herbarium. The team was particularly interested in documenting the presence of alien species in the two canyons.

\section{Chimenea Canyon Results}

Mark Fishbein, Shelley McMahon, George Ferguson, Victor Steinmann, and Amy Johnson conducted the research in Chimenea Canyon; Table 3 is the plant list resulting from the 1994 surveys. Surveyors counted 393 species representing 84 families. Nine species not listed in the Bowers and McLaughlin (1987) Rincon Mountains flora were observed, and thirteen alien species were recorded. Nine of the aliens were members of the grass family: Agrostis stolonifera, Avena fatua (wild oat), Bromus madritensis (red brome), Cynodon dactylon (Bermuda grass), Echinochloa colona, Eragrostis curvula var. conferta, E. lehmanniana (Lehmann lovegrass), Hordeum leporinum (barley), and Rhynchelytrum repens. Neither Pennisetum ciliare (buffel grass) nor P. setaceum (fountain grass) were encountered in Chimenea Canyon at the time of these studies. Other non-natives included Sonchus asper and S. oleraceus (Asteraceae), Cyperus esculentus (Cyperaceae), and Marrubium vulgare (Lamiaceae).

\section{Madrona Canyon Results}

Table 4 is the plant list for Madrona Canyon put together by Mark Fishbein. In this canyon, 290 species from 68 families were counted. "New" and non-native listings numbered ten each, with seven grasses in the non-native category. Unlike nearby Chimenea Canyon, Madrona Canyon hosted both buffel grass and fountain grass, non-natives recommended for removal by the Box Canyon study. 
Table 3. Chimenea Canyon Checklist of Vascular Plants. Chimenea Canyon lies within a Protected Natural Area within the Rincon Mountain District of Saguaro National Park. This list was compiled in 1994 by Mark Fishbein, Shelley McMahon, George Ferguson, Victor Steinmann, and Amy Johnson. Plant names follow Kartesz (1994).

$+=$ New addition to the flora of the Rincon Mountains of Bowers and McLaughlin (1987)

$*=$ Introduced species

\section{Acanthaceae}

Anisacanthus thurberi (Torrey) Gray

Carlowrightia arizonica Gray

Siphonoglossa longiflora (Torrey) Gray

Tetramerium nervosum Nees

\section{Agavaceae}

Agave cf. palmeri Engelmann

Agave schottii Engelmann var. schottii

Yucca schottii Engelmann

\section{Amaranthaceae}

Amaranthus albus L.

Gomphrena nitida Rothrock

Gomphrena sonorae Torrey

Iresine heterophylla Standley

\section{Amaryllidaceae}

Zephyranthes longifolia Hemsley

\section{Anacardiaceae}

Rhus trilobata Nuttall

Toxicodendron rydbergii (Small ex Rehder)

Greene

\section{Apiaceae}

Daucus pusillus Michaux

Spermolepis echinata (Nuttall ex DC.) Heller

\section{Apocynaceae}

Apocynum sp.

Haplophyton cimicidum DC.

\section{Araliaceae}

Aralia humilis Cavanilles

\section{Aristolochiaceae}

Aristolochia watsonii Wooton \& Standley

\section{Asclepiadaceae}

Asclepias linaria Cavanilles

Asclepias nyctaginifolia Gray

Asclepias tuberosa L. ssp. interior Woodson

Cynanchum arizonicum (Gray) Shinners

Matelea arizonica (Gray) Shinners

Sarcostemma cynanchoides Decaisne

ssp. hartwegii (Vail) R. Holm

\section{Aspleniaceae}

Woodsia cochisensis Windham

\section{Asteraceae}

Acourtia nana (Gray) Reveal \& King Acourtia thurberi (Gray) Reveal \& King Acourtia wrightii (Gray) Reveal \& King Ageratina paupercula (Gray) King \&

Robinson

Ambrosia ambrosioides (Cavanilles) Payne

Ambrosia confertiflora DC.

Artemisia ludoviciana Nuttall

ssp. albula (Wooton) Keck

Baccharis brachyphylla Gray

Baccharis salicifolia (Ruiz \& Pavón) Persoon

Baccharis sarothroides A. Gray

Baccharis thesioides Kunth

Bahia absinthifolia Bentham

var. dealbata (Gray) Gray

Bidens aurea (Aiton) Sherff

Bidens leptocephala Sherff

Brickellia amplexicaulis B.L. Robinson

Brickellia betonicifolia Gray

Brickellia californica (Torrey \& Gray) Gray

Brickellia coulteri Gray 
Brickellia venosa (Wooton \& Standley) B.L. Robinson Carminatia tenuiflora DC.

Cirsium neomexicanum Gray

Conyza canadensis (L.) Cronquist

Coreocarpus arizonicus (Gray) Blake

Cosmos parviflorus (Jacquin) Persoon

Encelia farinosa Gray ex Torrey

Ericameria laricifolia (Gray) Shinners

+ Erigeron colomexicanus A. Nelson

Erigeron divergens Torrey \& Gray

+ Erigeron neomexicanus Gray

Eupatorium solidaginifolium Gray

Gamochaeta purpurea (L.) Cabrera

Gnaphalium canescens DC.

Guardiola platyphylla Gray

Gymnosperma glutinosum (Sprengel)

Lessing

Heliomeris longifolia var. annua (Jones) Yates

Heterotheca subaxillaris (Lamarck) Britton

\& Rusby

Hieracium fendleri Schultz-Bipontinus

Hymenoclea monogyra Torrey \& Gray ex

Gray

Hymenothrix wrightii A. Gray

Isocoma tenuisecta Greene

Lactuca serriola L.

Lasthenia californica DC. ex Lindley

Machaeranthera asteroides (Torrey) Greene

Machaeranthera gracilis (Nuttall) Shinners

Machaeranthera pinnatifida (Hooker)

Shinners ssp. pinnatifida var. pinnatifida

Machaeranthera tagetina Greene

Microseris lindleyi (DC.) A. Gray

Pectis filipes Harvard \& Gray var. subnuda

Fernald

Perityle lemmoni (Gray) Macbride

Porophyllum gracile Bentham

Porophyllum ruderale (Jacquin) Cassini

ssp. macrocephalum (DC.) R.R. Johnson

Psilostrophe cooperi (Gray) Greene

Rafinesquia neomexicana Gray

Sanvitalia aberti Gray

Senecio lemmoni Gray
Senecio neomexicanus Gray

Solidago velutina $\mathrm{DC}$.

*Sonchus asper (L.) Hill

* Sonchus oleraceus L.

Stephanomeria pauciflora (Torrey) A.

Nelson

Tagetes lemmonii Gray

Tagetes micrantha Cavanilles

Trixis californica Kellogg

Viguiera dentata (Cavanilles) Sprengel

var. lancifolia Blake

Zinnia acerosa (DC.) Gray

Berberidaceae

Berberis wilcoxii Kearney

\section{Betulaceae}

Alnus oblongifolia Torrey

\section{Boraginaceae}

Cryptantha barbigera (Gray) Greene

Cryptantha micrantha (Torrey) I.M. Johnston

Cryptantha muricata (Hooker \& Arnott)

Nelson \& Macbride

var. denticulata (Greene) I.M. Johnston

Cryptantha nevadensis A. Nelson \&

Kennedy

Harpogonella palmeri Gray

Pectocarya platycarpa (Munz \& Johnston)

Munz \& Johnston

Pectocarya recurvata I.M. Johnston

Plagiobothrys arizonicus (Gray) Greene ex

Gray

\section{Brassicaceae}

Arabis perennans S. Watson

Descurainia pinnata (Walter) Britton

Lepidium virginicum $\mathrm{L}$. var. medium

(Greene)

C.L. Hitchcock

Lesquerella gordonii (Gray) S. Watson 
Schoenocrambe linearifolia (Gray) Rollins Thysanocarpus curvipes Hooker

\section{Cactaceae}

Carnegia gigantea (Engelmann) Britton \&

Rose

Echinocereus coccineus Engelmann

Echinocereus fasciculatus (Engelmann) L.

Benson

var. fasciculatus [probably E. fendleri

(Engelmann) F. Seitz var. fasciculatus

(Engelmann ex B.D. Jackson) N. P.

Taylor]

Echinocereus rigidissimus (Engelmann)

Haage $\mathrm{f}$.

Ferocactus wislizenii (Engelmann) Britton \& Rose

Mammillaria grahamii Engelmann

[incl. M. g. var. oliviae (Orcutt) Benson, M. microcarpa Engelmann]

Mammillaria viridiflora (Britton \& Rose)

Böcker

Opuntia bigelovii Engelmann

Opuntia chlorotica Engelmann \& Bigelow

Opuntia engelmannii Salm-Dyck

Opuntia fulgida Engelmann var. fulgida

Opuntia fulgida Engelmann

var. mammillata (Schott) Coulter

Opuntia phaeacantha Engelmann

Opuntia spinosior (Engelmann) Toumey

Opuntia versicolor Engelmann

\section{Campanulaceae}

Triodanis perfoliata (L.) Nieuwland var. biflora (Ruiz \& Pavón)

Triodanis perfoliata (L.) Nieuwland

\section{Caprifoliaceae}

Symphoricarpos oreophilus A. Gray

\section{Caryophyllaceae}

Cerastium texanum Britton

Drymaria molluginea (Lagasca) Didrichsen

Silene antirrhina $\mathrm{L}$.

\section{Chenopodiaceae}

Atriplex canescens (Pursh) Nuttall

+ Chenopodium neomexicanum Standley

\section{Commelinaceae}

Commelina dianthifolia Delile

Commelina erecta $\mathrm{L}$.

\section{Convolvulaceae}

Evolvulus alsinoides (L.) L. Ipomoea barbatisepala A. Gray

Ipomoea costellata Torrey Ipomoea cristulata $\mathrm{H}$. Hallier

Ipomoea purpurea (L.) Roth

Crassulaceae

Crassula erecta (Hooker \& Arnott) Berger

Crossosomataceae

Crossosoma bigelovii $\mathrm{S}$. Watson

Cucurbitaceae

Marah gilensis Greene

\section{Cupressaceae}

Juniperus deppeana Steudel

\section{Cyperaceae}

Carex chihuahuensis Mackenzie

Carex senta Boott

Cyperus dipsaceus Liebmann

*Cyperus esculentus L.

Cyperus mutisii (Kunth) Grisebach

[incl. C. pringlei Britton]

Cyperus pallidicolor (Kükenthal) G.C.

Tucker

[C. flavus (Vahl) Nees, misapplied]

Cyperus squarrosus L.]

Eleocharis montevidensis Kunth

Lipocarpha micrantha (Vahl) G. Tucker

\section{Ericaceae}

Arbutus arizonica (A. Gray) Sargent

Arctostaphylos pungens H.B.K. 
Euphorbiaceae

Acalypha neomexicana Mueller-Argoviensis

Ditaxis neomexicana (Mueller-Argoviensis)

Heller

Chamaesyce capitellata (Engelmann)

Millspaugh

Euphorbia heterophylla L.

Euphorbia hyssopifolia L.

Euphorbia melanadenia Torrey

Euphorbia pediculifera Engelmann

Jatropha cardiophylla (Torrey) Mueller-

Argoviensis

Tragia nepetifolia Cavanilles

\section{Fabaceae}

Acacia angustissima (Millspaugh) Kuntze

Acacia greggii A. Gray

Amorpha fruticosa L.

Astragalus allochrous A. Gray

Astragalus arizonicus A. Gray

Astragalus nuttallianus A. DC.

Calliandra eriophylla Bentham

Calliandra humilis Bentham

Coursetia caribaea (Jacquin) Lavin var. sericea Gray) Lavin

Coursetia glandulosa A. Gray

Crotalaria pumila Ortega

Dalea albiflora A. Gray

Dalea pringlei A. Gray

Dalea pulchra Gentry

Dalea versicolor Zuccarini ssp. versicolor var. sessilis (A. Gray) Barneby

Desmodium batocaulon A. Gray

Desmodium cinerascens A. Gray

Desmodium rosei Schubert

Erythrina flabelliformis Kearney

Eysenhardtia orthocarpa (A. Gray) S.

Watson

Galactia wrightii A. Gray

Lotus greenei Ottley ex Kearney \& Peebles

Lotus humistratus Greene

Lotus oroboides (Kunth) Ottley

var. nanus (A. Gray) Isely

Lotus oroboides (Kunth) Ottley var.

oroboides
Lupinus concinnus Agardh

Lupinus sparsiflorus Bentham

Lysiloma watsoni Rose

Macroptilium gibbosifolium (Ortega) A.

Delgado

Mimosa aculeaticarpa Ortega

var. biuncifera (Bentham) Barneby

Parkinsonia florida (Bentham ex A. Gray) S.

Watson

Parkinsonia microphylla Torrey

Phaseolus acutifolius A. Gray

Prosopis velutina Wooton

Robinia neomexicana A. Gray

Tephrosia leiocarpa A. Gray

Tephrosia vicioides Schlechtendal [T. tenella

A. Gray]

Vicia ludoviciana Nuttall

Fagaceae

Quercus arizonica Sargent

Quercus emoryi Torrey

Quercus hypoleucoides Camus

Quercus oblongifolia Torrey

Fouquieriaceae

Fouquieria splendens Engelmann ssp.

splendens

Garryaceae

Garrya wrightii Torrey

Geraniaceae

Erodium cicutarium (L.) Héritier

Geranium caespitosum James

Geranium carolinianum L.

Hydrophyllaceae

Phacelia distans Bentham

Phacelia sp.

Iridaceae

Sisyrinchium cernuum (Bicknell) Kearney

Juncaceae

Juncus acuminatus Michaux

Juncus bufonius L. 
Juncus marginatus Rostkovius

Juncus tenuis Willdenow [J. interior Wieg.]

Juglandaceae

Juglans major (Torrey) Heller

Krameriaceae

Krameria grayi Rose \& Painter?

$\{K$. parvifolia Bentham?\}

Lamiaceae

Hedeoma nana (Torrey) Briquet ssp. nana

Hyptis emoryi Torrey

* Marrubium vulgare L.

Monarda citriodora Cervantes ex Lagasca ssp. austromontana (Epling) Scora

Stachys coccinea Jacquin

\section{Liliaceae}

Dichelostemma pulchellum (Salisbury) Heller

Loasaceae

Mentzelia asperula Wooton \& Standley

\section{Lythraceae}

Cuphea wrightii A. Gray

Malpighiaceae

Janusia gracilis A. Gray

\section{Malvaceae}

Abutilon abutiloides (Jacquin) Garcke

Abutilon incanum (Link) Sweet

Abutilon mollicomum (Willdenow) Sweet

Anoda abutiloides A. Gray

Anoda cristata (L.) Schlechtendal

Gossypium thurberi Todaro

Herissantia crispa (L.) Brizicky

Hibiscus coulteri Harvey

Hibiscus denudatus Bentham

Rhynchosida physocalyx (A. Gray) Fryxell

Sphaeralcea fendleri A. Gray

Sphaeralcea laxa Wooton \& Standley

\section{Moraceae}

Morus microphylla Buckley

Nolinaceae

Dasylirion wheeleri S. Watson

Nolina microcarpa S. Watson

Nyctaginaceae

Allionia incarnata $\mathrm{L}$.

Boerhaavia diffusa $\mathrm{L}$.

Boerhaavia scandens $\mathrm{L}$.

Mirabilis coccineus (Torrey) Bentham \&

Hooker $\mathrm{f}$.

Mirabilis longiflora $\mathrm{L}$.

Mirabilis oblongifolia (A. Gray) Heimerl

Oleaceae

Fraxinus velutina Torrey

Menodora scabra (Engelmann) A. Gray

Onagraceae

Epilobium canum (Greene) Raven

ssp. latifolium (Hooker) Raven

Oenothera elata Kunth. ssp. hirsutissima

(Gray ex. S. Wats.)

\section{Oxalidaceae}

Oxalis albicans H.B.K. ssp. pilosa (Nuttall)

Eiten

Oxalis alpina (Rose) Knuth

Papaveraceae

Corydalis aurea Willdenow

\section{Phytolaccaceae}

Rivina humilis L.

\section{Pinaceae}

Pinus cembroides Zuccarini

Pinus leiophylla Schiede \& Deppe var. chihuahuana (Engelmann) Shaw

Pinus ponderosa $\mathrm{P} \& \mathrm{C}$ var. scopulorum Engelmann 
Plantaginaceae

Plantago patagonica Jacquin

Plantago virginica $\mathrm{L}$.

Platanaceae

Platanus wrightii S. Watson

Plumbaginaceae

Plumbago scandens L.

Poaceae

+ Aegopogon tenellus (DC.) Trinius

*Agrostis stolonifera L.

Aristida adscencionis L.

Aristida havardii Vasey

Aristida ternipes Cavanilles var. ternipes

* Avena fatua $\mathrm{L}$.

Bothriochloa barbinodis (Lagasca) Herter

Bouteloua aristidoides (H.B.K.) Grisebach

Bouteloua barbata Lagasca

Bouteloua curtipendula (Michaux) Torrey

Bouteloua hirsuta Lagasca

Bouteloua repens (H.B.K.) Scribner \&

Merrill

Bouteloua rothrockii Vasey

Bromus carinatus Hooker \& Arnott

*Bromus madritensis L.

* Cynodon dactylon (L.) Persoon

Digitaria californica Henrard

*Echinochloa colona (L.) Link

Elymus elymoides (Rafinesque) Swezey

Elyonurus barbiculmis Hackel

* Eragrostis cilianensis (All.)

Vignolo-Lutati ex Janchen

*+Eragrostis curvula (Schrad.) Nees var. conferta Stapf.

Eragrostis intermedia Hitchcock

* Eragrostis lehmanniana Nees

Eragrostis mexicana (Hornemann) Link ssp. mexicana

Eragrostis pectinacea (Michaux) Nees var. pectinacea

Eragrostis pectinacea (Michaux) Nees var. miserrima (Fournier) J. Reeder

Eriochloa acuminata (Presl) Kunth var. acuminata

Eriochloa aristata Vasey

Erioneuron pulchellum (H.B.K.) Tateoka

Heteropogon contortus (L.) Beauvois

ex Roemer \& Schultes

Heteropogon melanocarpus (Elliot) Elliot ex Bentham

Hilaria belangeri (Steudel) Nash

*Hordeum murinum L. ssp. leporinum (Link) Arcang.

Leptochloa dubia (H.B.K.) Nees

Lycurus setosus (Nuttall) C. Reeder

Muhlenbergia emersleyi Vasey

Muhlenbergia minutissima (Steudel) Swallen

Muhlenbergia pauciflora Buckley

Muhlenbergia porteri Scribner

Muhlenbergia rigens (Bentham) Hitchcock

Muhlenbergia sinuosa Swallen

Muhlebergia texana Buckley

Panicum bulbosum H.B.K.

Panicum hirticaule Presl

Dicanthelium oligosanthes (Schultes) Gould

var. scriberianum (Nash) Gould

Piptochaetium fimbriatum (Kunth) Hitchcock

Polypogon monspeliensis (L.) Desfontaines

* Rhynchelytrum repens (Willdenow) C.E.

Hubbard

Schizachyrium cirratum (Hackel) Wooton \&

Standley

Schizachyrium sanguineum (Retzius) Alston

Setaria grisebachii Fournier

Sporobolus contractus A.S. Hitchcock

Trachypogon secundus (Presl.) Scribner

Tridens muticus (Torrey) Nash

Vulpia microstachys (Nuttall) Munro

var. ciliata (Beal) Lonard \& Gould

Vulpia octoflora (Walter) Rydberg var.

octoflora

\section{Polemoniaceae}

Eriastrum diffusum (A. Gray) Mason

Ipomopsis multiflora (Nuttall) V. Grant

Linanthus aureus (Nuttall) Greene 


\section{Polygalaceae}

Monnina wrightii A. Gray

Polygala alba Nuttall

Polygala obscura Bentham

Polygonaceae

Eriogonum wrightii Torrey var. wrightii

Rumex crispus L.

\section{Portulacaceae}

Calandrinia ciliata (Ruiz \& Pavon) DC.

Portulaca suffrutescens Engelmann

Portulaca umbraticola Kunth

ssp. lanceolata (Engelmann) Matthews \&

Ketron

Talinum paniculatum (Jacquin) Gaertner

\section{Primulaceae}

+ Centunculus minimus L.

Psilotaceae

+ Psilotum nudum (L.) Beauvois

\section{Pteridaceae}

Astrolepis cochisensis (Goodding) Benham $\&$

Windham [Notholaena cochisensis

Goodding]

Astrolepis sinuata (Lagasca) Benham \&

Windham

[Notholaena sinuata (Lagasca) Kaulfuss]

Bommeria hispida (Metternich) Underwood

Cheilanthes bonariensis (Willdenow) Proctor

[Notholaena aurea (Poiret) Desvaux]

+ Cheilanthes covillei Maxon

Cheilanthes lindheimeri Hooker

Cheilanthes wrightii Hooker

Notholaena lemmonii D.C. Eaton

Notholaena standleyi Maxon

Pellaea truncata Goodding

Pellaea wrightiana Hooker

Pentagramma triangularis (Kaulfuss)

Yatskievych,

Windham \& Wollenweber ssp. maxonii

(Weatherby) Yatskievych, Windham \&
Wollenweber [Pityrogramma triangularis (Kaulfuss) Maxon var.

maxonii Weatherby]

\section{Ranunculaceae}

Anemone tuberosa Rydberg

Aquilegia chrysantha Gray

Delphinium scaposum Greene

\section{Rhamnaceae}

Condalia correllii M.C. Johnston

Condalia warnockii M.C. Johnston

var. kearneyana M.C. Johnston

Rhamnus californica Eschscholtz

Rhamnus crocea Nuttall

ssp. ilicifolia (Kellogg) C.B. Wolf

Ziziphus obtusifolia (Hooker) A. Gray

Rosaceae

Prunus serotina Ehrgardt

ssp. virens (Wooton \& Standley)

McVaugh

Vauquelinia californica (Torrey) Sargent

\section{Rubiaceae}

Bouvardia ternifolia (Cavanilles)

Schlechtendal

Galium aparine L.

Galium mexicanum Kunth

ssp. asperrimum (Gray) Dempster

Galium microphyllum Gray

Galium proliferum Gray

Galium wrightii A. Gray

\section{Salicaceae}

Populus fremontii S. Watson ssp. fremontii

Salix exigua Nuttall

Salix gooddingii Ball

\section{Santalaceae}

Commandra umbellata (L.) Nuttall

\section{Sapindaceae}

Dodonea viscosa (L.) Jacquin

Sapindus drummondii Hooker \& Arnott 


\section{Sapotaceae}

Sideroxylon lanuginosa Michaux [Bumelia lanuginose

(Michaux) Persoon var. rigida A. Gray]

\section{Saxifragaceae}

Heuchera sanguinea Engelmann

\section{Scrophulariaceae}

Antirrhinum nuttallianum Bentham

Brachystigma wrightii (Gray) Pennell

Castilleja minor (Gray) Gray

Castilleja tenuiflora Bentham

Linaria texana Scheele

Maurandya antirrhiniflora Humboldt \&

Bonpland

ex Willdenow

Mecardonia vandellioides (H.B.K.) Pennell

Mimulus guttatus DC.

Mimulus nasutus Greene

Penstemon parryi (A. Gray) A. Gray

Veronica peregrina $\mathrm{L}$.

\section{Selaginellaceae}

Selaginella rupincola Underwood

\section{Solanaceae}

Lycium berlandieri Dunal

Lycium exsertum Gray

Nicotiana trigonophylla Dunal

Physalis cf. hederaefolia Gray

Solanum eleagnifolium Cavanilles

\section{Sterculiaceae}

Ayenia compacta Rose

\section{Typhaceae}

Typha domingensis Persoon

\section{Ulmaceae}

Celtis pallida Torrey

Celtis reticulata Torrey

Urticaceae

Parietaria hespera Hinton

Verbenaceae

Aloysia wrightii A.A. Heller ex Abrams

Glandularia bipinnatifida (Nuttall) Nuttall

var. bipinnatifida

\section{Viscaceae}

Phoradendron californicum Nuttall

Phoradendron coryae Trelease $[P$. villosum

(Nuttall)

Nuttall ssp. coryae (Trelease) Wiens]

+ Phoradendron juniperinum Engelmann

Phoradendron macrophyllum (Engelmann)

Cockerell

Vitaceae

Vitis arizonica Engelmann 
Table 4. Madrona Canyon Checklist of Vascular Plants. Madrona Canyon lies within a Protected Natural Area within the Rincon Mountain District of Saguaro National Park. This list was compiled in 1995 by Mark Fishbein. Plant names follow Kartesz (1994).

$+=$ New addition to the flora of the Rincon Mountains of Bowers and McLaughlin (1987)

$*=$ Introduced species

\section{Acanthaceae}

Anisacanthus thurberi (Torrey) A. Gray

Siphonoglossa longiflora (Torrey) A. Gray

Tetramerium nervosum Nees

\section{Agavaceae}

Agave chrysantha Peebles

Agave schottii Engelmann

Yucca schottii Engelmann

\section{Amaranthaceae}

Amaranthus albus L.

Gomphrena sonorae Torrey

Iresine heterophylla Standley

\section{Anacardiaceae}

Toxicodendron rydbergii (Small ex Rydberg)

Greene

\section{Apiaceae}

Daucus pusillus Michaux

Spermolepis echinata (Nuttall) Heller

\section{Apocynaceae}

Haplophyton crooksii L.

\section{Araliaceae}

Aralia humilis Cavanilles

\section{Aristolochiaceae}

Aristolochia watsonii Wooton \& Standley

\section{Asclepiadaceae}

Asclepias glaucescens Kunth

Asclepias linaria Cavanilles

Gonolobus arizonicus (Gray) Woodson

Sarcostemma cynanchoides Decaisne ssp. hartwegii (Vail) R. Holm

\section{Asteraceae}

Acourtia thurberi (A. Gray) Reveal \& King Ageratina paupercula (A. Gray) King \&

Robinson

Ambrosia ambrosioides (Cavanilles) Payne

Ambrosia confertiflora DC.

Artemisia ludoviciana Nuttall

Baccharis salicifolia (Ruiz \& Pavon) Persoon

Baccharis sarothroides A. Gray

Baccharis thesioides H.B.K.

Bebbia juncea (Bentham) Greene

Bidens aurea (Aiton) Sherff

Brickellia amplexicaulis B.L. Robinson

Brickellia betonicifolia A. Gray

Brickellia californica (Torrey \& A. Gray) A.

Gray

Brickellia coulteri A. Gray var. coulteri

Brickellia venosa (Wooton \& Standley) B.L.

Robinson

Carminatia tenuiflora DC.

Cirsium neomexicanum A. Gray

Coreocarpus arizonicus (A. Gray) Blake

Encelia farinosa A. Gray ex Torrey

Ericameria laricifolia (A. Gray) Shinners

Erigeron divergens Torrey \& A. Gray

Erigeron neomexicanus A. Gray

Eupatorium solidaginifolium A. Gray

Gamochaeta purpurea (L.) Cabrera

Gnaphalium leucocephalum A. Gray

Gnaphalium wrightii A. Gray

Guardiola platyphylla A. Gray

Gutierrezia microcephala (DC.) A. Gray

Gymnosperma glutinosum (Sprengel)

Lessing

Hymenothrix wrightii A. Gray 
Isocoma tenuisecta Greene

Lasianthaea podocephala (A. Gray) K.

Becker

[Zexmenia podocephala A. Gray]

Machaeranthera asteroides (Torrey) Greene

[M. tephrodes (A. Gray) Greene]

Machaeranthera gracilis (Nuttall) Shinners

Machaeranthera tagetina Greene

+Melampodium longicorne A. Gray

+Pectis cylindrica (Fernald) Rydberg

Perityle lemmonii (A. Gray) Macbride

Porophyllum macrocephalum DC. [P. ruderale (Jacq.)

Cass. ssp. macrocephalum (DC.) R.R. Johnson]

Sanvitalia abertii A. Gray

Solidago velutina DC.

*Sonchus oleraceus L.

Stephanomeria pauciflora (Torrey) A. Nelson

Trixis californica Kellogg

Viguiera annua (Jones) Blake

Viguiera dentata (Cavanilles) Sprengel var. lancifolia Blake

Zinnia acerosa (DC.) A. Gray

\section{Betulaceae}

Alnus oblongifolia Torrey

\section{Bignoniaceae}

Tecoma stans (L.) Juss. ex Kunth

\section{Brassicaceae}

Schoenocrambe linearifolia (A. Gray)

Rollins

\section{Cactaceae}

Carnegia gigantea (Engelmann) Britton \& Rose

Echinocereus coccineus Engelmann

Echinocereus fasciculatus (Engelmann) L.

Benson

var.fasciculatus [probably E. fendleri

(Engelmann) F. Seitz var. fasciculatus
(Engelmann ex B.D. Jackson) N. P .

Taylor]

Echinocereus rigidissimus (Engelmann)

Haage $\mathrm{f}$.

Ferocactus wislizenii (Engelmann) Britton \& Rose

Mammillaria grahamii Engelmann [incl. var. oliviae

(Orcutt) Benson, M. microcarpa

Engelmann]

Opuntia chlorotica Engelmann \& Bigelow

Opuntia engelmannii Salm-Dyck

[O. phaeacantha Engelmann

var. discata (Griffiths) Benson \&

Walkington]

Opuntia fulgida Engelmann var. fulgida

Opuntia fulgida Engelmann

var. mammillata (Schott) Coulter

Opuntia phaeacantha Engelmann

[O. p. var. major Engelmann]

Opuntia spinosior (Engelmann) Toumey

Opuntia versicolor Engelmann

\section{Campanulaceae}

Triodanis sp.

\section{Caryophyllaceae}

Drymaria molluginea (Lagasca) Didrichsen

Silene antirrhina $\mathrm{L}$.

\section{Chenopodiaceae}

Atriplex canescens (Pursh) Nuttall

Chenopodium neomexicanum Standley

[C. palmeri Standley]

\section{Cochlospermaceae}

Amoreuxia palmatifida Moçino \& Sessé 
Commelinaceae

Commelina dianthifolia Delile

Commelina erecta L.

\section{Convolvulaceae}

Evolvulus alsinoides (L.) L. var. angustifolius

Torrey

Evolvulus arizonicus A. Gray

Ipomoea barbatisepala A. Gray

Ipomoea costellata Torrey

Ipomoea cristulata $\mathrm{H}$. Hallier $\mathrm{f}$.

Ipomoea leptotoma Torrey

\section{Crossosomataceae}

Crossosoma bigelovii S. Watson

\section{Cucurbitaceae}

Echinopepon wrightii (A. Gray) S. Watson

\section{Cupressaceae}

Juniperus deppeana Steudel

\section{Cyperaceae}

+Bulbostylis funckii (Steudel) C.B. Clarke

* Cyperus esculentus L.

Cyperus mutisii (H.B.K.) Grisebach

[incl. C. pringlei Britton]

Cyperus pallidicolor (Kükenthal) G. Tucker

[C. flavus (Vahl) Nees, misapplied]

Cyperus squarrosus L. [C. aristatus Rottbøll]

Lipocarpha micrantha (Vahl) G. Tucker

[Hemicarpha micrantha (Vahl) Britton]

\section{Dryopteridaceae}

Woodsia cochisensis Windham

[W. plummerae Lemmon, in part]

\section{Euphorbiaceae}

Acalypha neomexicana Mueller-Argoviensis Chamaesyce florida (Engelmann) Millspaugh Euphorbia heterophylla L.

Chamaesyce hyssopifolia (L.) Standley

Chamaesyce melanadenia (Torrey)

Millspaugh

Chamaesyce pediculifera (Engelmann)
Rose \& Standley

Jatropha cardiophylla (Torrey) Mueller-

Argoviensis

Tragia nepetifolia Cavanilles

\section{Fabaceae}

Acacia angustissima (Miller) Kuntze

Acacia greggii A. Gray

Amorpha fruticosa L.

Astragalus nothoxys Gray

Calliandra eriophylla Bentham

Chamaecrista nictitans (L.) Greene

var. mensalis (Greenman) Irwin \&

Barneby

Cologania angustifolia H.B.K.

Coursetia caribaea (Jacquin) Lavin

var. sericea (Gray) Lavin

Coursetia glandulosa Gray

Crotalaria pumila Ortega

Dalea albiflora Gray

Dalea pringlei Gray

Dalea filiformis Gray

Desmodium batocaulon Gray

Desmodium cinerascens Gray

Desmodium grahamii Gray

Desmodium procumbens (Miller) A.S.

Hitchcock

var. exiguum (Gray) Schubert

Erythrina flabelliformis Kearney

Eysenhardtia orthocarpa (Gray) S. Watson

Galactia wrightii Gray

Lathyrus graminifolius (S. Watson) White

Lysiloma watsoni Rose

Macroptilium gibbosifolium (Ortega)

Delgado

Mimosa aculeaticarpa Ortega

var. biuncifera (Bentham) Barneby

Parkinsonia florida (Bentham ex Gray) S.

Watson

Phaseolus acutifolius Gray

Phaseolus maculatus Scheele

Prosopis velutina Wooton

Senna covesii (Gray) Irwin \& Barneby

Senna hirsuta (L.) Irwin \& Barneby 
var glaberrima (M.E. Jones) Irwin \&

Barneby

Tephrosia leiocarpa Gray

\section{Fagaceae}

Quercus arizonica Sargent

Quercus emoryi Torrey

Quercus hypoleucoides Camus

Quercus oblongifolia Torrey

\section{Fouquieriaceae}

Fouquieria splendens Engelmann ssp. splendens

\section{Hydrangeaceae}

Philadelphus microphyllus A. Gray

[Philadelphus argenteus Rydberg]

\section{Juncaceae}

Juncus acuminatus Michaux

+ Juncus balticus Willdenow

Juncus tenuis Willdenow [J. interior Wieg.]

\section{Juglandaceae}

Juglans major (Torrey) Heller

\section{Lamiaceae}

Hedeoma dentata Torrey

Hyptis emoryi Torrey

+Salvia reflexa Hornemann

Stachys coccinea Jacquin

\section{Loasaceae}

Mentzelia asperula Wooton \& Standley

\section{Malpighiaceae}

Janusia gracilis A. Gray

\section{Malvaceae}

Abutilon abutiloides (Jacquin) Garcke [A. californicum Bentham]

Abutilon incanum (Link) Sweet

Abutilon mollicomum (Willdenow) Sweet

[A. sonorae A. Gray]
Anoda abutiloides A. Gray

Anoda cristata (L.) Schlechtendal

Gossypium thurberi Todaro

Hibiscus coulteri Harvey

Martyniaceae

Proboscidea parviflora (Wooton) Wooton \&

Standley

var. parviflora

\section{Moraceae}

Morus microphylla Buckley

\section{Nolinaceae}

Dasylirion wheeleri S. Watson

Nolina microcarpa S. Watson

\section{Nyctaginaceae}

Allionia incarnata L.

Boerhavia diffusa $\mathrm{L}$.

Boerhavia scandens L.

Mirabilis coccinea (Torrey) Bentham \&

Hooker

Mirabilis longiflora L.

\section{Oleaceae}

Fraxinus velutina Torrey

Onagraceae

Epilobium canum (Greene) Raven

ssp. latifolium (Hooker) Raven

Oenothera elata H.B.K. ssp. hirsutissima

(A. Gray) Dietrich [Oe. hookeri Torrey \& A. Gray]

\section{Oxalidaceae}

Oxalis albicans Kunth ssp. pilosa (Nuttall)

Eiten

Oxalis alpina (Rose) Knuth

\section{Phytolaccaceae}

Rivina humilis L.

Pinaceae

Pinus cembroides Zuccarini

Pinus leiophylla Schiede \& Deppe 
var. chihuahuana (Engelmann) Shaw

\section{Plantaginaceae}

Plantago virginica L.

Plumbaginaceae

Plumbago scandens L.

\section{Poaceae}

(Verified J.R. \& C.R. Reeder)

+ Aegopogon tenellus (DC.) Trinius

Agrostis scabra Willdenow

Aristida adscencionis L.

Aristida schiedeana Trinius \& Ruprecht

var. orcuttiana (Vasey) Allred \& Valdes

$\mathrm{R}$.

Aristida ternipes Cavanilles var. ternipes

Bothriochloa barbinodis (Lagasca) Herter

Bouteloua aristidoides (H.B.K.) Grisebach

Bouteloua barbata Lagasca

Bouteloua curtipendula (Michaux) Torrey

Bouteloua hirsuta Lagasca

Bouteloua repens (H.B.K.) Scribner \&

Merrill

Brachiaria arizonica (Scribner \& Merrill)

S.T. Blake

*Bromus madritensis L.

* Cynodon dactylon (L.) Persoon

Digitaria californica Henrard

${ }^{*}$ Echinochloa crusgalli (L.) Beauvois

Elyonurus barbiculmis Hackel

Eragrostis intermedia Hitchcock

*Eragrostis lehmanniana Nees

Eragrostis mexicana (Hornemann) Link ssp. mexicana

Eragrostis pectinacea (Michaux) Nees var. pectinacea

Eriochloa aristata Vasey

Heteropogon contortus (L.) Beauvois

ex Roemer \& Schultes

Heteropogon melanocarpus (Elliot) Elliot ex

Bentham

Leptochloa dubia (Kunth) Nees

Leptochloa mucronata (Michaux) Kunth

Lycurus setosus (Nuttall) C. Reeder
Muhlenbergia dumosa Scribner

Muhlenbergia emersleyi Vasey

Muhlenbergia fragilis Swallen

Muhlenbergia monticola Buckley

Muhlenbergia pauciflora Buckley

Muhlenbergia porteri Scribner

Muhlenbergia rigens (Bentham) Hitchcock

Muhlenbergia sinuosa Swallen

Panicum bulbosum Kunth

Panicum hirticaule J. Presl

Dicanthelium oligosanthes (Schult.) Gould var. scribnerianum (Nash) Gould

+*Pennisetum ciliare (L.) Link

+*Pennisetum setaceum (Forskål) Chiov.

Piptochaetium fimbriatum (H.B.K.)

Hitchcock

* Rhynchelytrum repens (Willdenow) C.E.

Hubbard

Schizachyrium sanguineum (Retzius) Alston

Setaria macrostachya Kunth

Trachypogon secundus (J. Presl) Scribner

\section{Polygalaceae}

Monnina wrightii A. Gray

Polygala obscura Bentham

Polygonaceae

Eriogonum wrightii Torrey var. wrightii

Polygonum hydropiperoides Michaux

*Rumex crispus L.

\section{Portulacaceae}

Portulaca oleracea L. [P. retusa Engelmann] Portulaca suffrutescens Engelmann

Portulaca umbraticola Kunth ssp. lanceolata (Engelmann) Matthews \& Ketron 


\section{Pteridaceae}

Astrolepis sinuata (Lagasca) Benham \&

Windham

[Notholaena sinuata (Lagasca) Kaulfuss]

Bommeria hispida (Metternich) Underwood

Cheilanthes bonariensis (Willdenow) Proctor

[Notholaena aurea (Poiret) Desvaux]

+ Cheilanthes covillei Maxon

Cheilanthes lindheimeri Hooker

Cheilanthes wootonii Maxon

Cheilanthes wrightii Hooker

Cheilanthes yavapensis T. Reeves ex

Windham

[C. wootonii Maxon, in part]

Notholaena grayi Davenport

Notholaena lemmonii D.C. Eaton

Notholaena standleyi Maxon

Pellaea truncata Goodding

Pellaea wrightiana Hooker

\section{Ranunculaceae}

Aquilegia chrysantha A. Gray

Clematis ligusticifolia Nuttall

\section{Rhamnaceae}

Condalia warnockii M.C. Johnston var. kearneyana M.C. Johnston

Rhamnus crocea Nuttall

ssp. ilicifolia (Kellogg) C.B. Wolf

Ziziphus obtusifolia (Hooker) A. Gray

\section{Rosaceae}

Vauquelinia californica (Torrey) Sargent

\section{Rubiaceae}

Bouvardia ternifolia (Cavanilles)

Schlechtendal

Diodia teres Walter

Galium mexicanum H.B.K. ssp. asperrimum (Gray) Dempster

Galium microphyllum Gray

Galium wrightii Gray
Ptelea trifoliata L.

Salicaceae

Populus fremontii S. Watson ssp. fremontii

Salix exigua Nuttall

Salix gooddingii Ball

Sapindaceae

Sapindus drummondii Hooker \& Arnott

Saxifragaceae

Heuchera sanguinea Engelmann

Scrophulariaceae

Castilleja tenuiflora Bentham

Schistophragma intermedia (Gray) Pennell

Mecardonia vandellioides (Kunth) Pennell

Mimulus guttatus DC.

Mimulus nasutus Greene

Penstemon parryi (A. Gray) A. Gray

Stemodia durantifolia (L.) Swartz

Selaginellaceae

Sellaginella rupincola Underwood

\section{Solanaceae}

Lycium berlandieri Dunal

Lycium exsertum Gray

Physalis sp.

\section{Sterculiaceae}

Ayenia compacta Rose

Typhaceae

Typha dominguensis Persoon

Verbenaceae

Aloysia wrightii A. Heller ex Abrams

Glandularia bipinnatifida (Nuttall) Nuttall var. bipinnatifida

Rutaceae 
Viscaceae

Phoradendron californicum Nuttall

Phoradendron coryae Trelease

Phoradendron macrophyllum (Engelmann)

Cockrum

\section{Vitaceae}

Cissus trifoliata L.

Vitis arizonica Engelmann 


\title{
Wildhorse Canyon (Rincon Mountain District)
}

\author{
By Renée Rondeau and Rebecca Van Devender
}

A portion of Wildhorse Canyon, approximately 194 ha (420 ac), as defined by T14S, R16E, SW $1 / 4$ and $\mathrm{S}^{1} / 2$ of SE $1 / 4$ of Section 14 , and $\mathrm{E}^{1 / 2}$ of $\mathrm{NW}^{1} / 4$ and $\mathrm{NE}^{1} / 4$ of Section 23 has been designated as a Protected Natural Area within the Rincon Mountain District of Saguaro National Park (Figure 2). This unique desert riparian habitat consists of a deep tinaja near the mouth of an ephemeral stream canyon. The tinaja holds an estimated minimum 0.5 acre-feet of water at capacity.

Subsequent to a fire in 1989 the tinaja silted in; at publication time (fall, 2000), the tinaja remains so. However, several smaller basins farther upstream hold enough water to maintain year-round shallow pools. The riparian corridor allows for a remarkably rich flora and makes this a prime wildlife area. Wildlife benefit from the water during drought periods, and park visitors enjoy viewing wildlife and the beauty and tranquility of the area.

A two-mile trail allows easy access to the tinaja by hikers and horseback riders. Presently the horses are allowed to be hitched to the tinaja itself, putting excess burden on a small area. Horses trample the native vegetation, leave wastes in or near the tinaja, and bring seeds of introduced species. Few visitors proceed beyond the tinaja where there is no trail and the canyon becomes choked with vegetation. However, disturbance in the lower part of the canyon has resulted in the migration of exotics upstream.

We conducted a floristic survey of the PNA in the spring (April 16 and 17) and fall (September 25, 26, and 27 ) of 1992, with a return trip by Van Devender and David Bertelsen on May 13. Figure 2 shows the transects surveyed. Three hundred-sixty species in 72 families were identified (Table 5). An elevational range of approximately $460 \mathrm{~m}(1500 \mathrm{ft})$ within the PNA accounts for much of this diversity. However, the canyon itself supports a large number of species. The cool, mesic streambed as well as shady rock shelves and niches in steep canyon walls provide habitat for many plants that would otherwise not be able to grow at such low elevations.

\section{Vegetation}

The area supports four vegetation types: 1) Saguaro-Foothills Paloverde Desertscrub, 2) Desert Riparian, 3) Desert-grassland, and 4) Juniper-Oak Woodland.

\section{Saguaro-Foothills Paloverde Desertscrub}

This desertscrub is the prevalent association for much of the proposed PNA, occurring approximately 920-1250 m (3000-4100 ft) elevation on slopes and ridges. It is a diverse Arizona Upland community within the Sonoran Desert, characterized by large numbers of cacti and other drought-tolerant trees and shrubs. The dominant overstory plants are saguaro (Carnegia gigantea), foothills paloverde (Parkinsonia microphylla), mesquite (Prosopis velutina), whitethorn acacia, (Acacia constricta), and ocotillo (Fouquieria splendens). Terpentine bush (Ericameria laricifolia) and fairy duster (Calliandra eriophylla) are common subshrubs. Brittle bush (Encelia farinosa) is especially common on south slopes while sotol (Dasylirion wheeleri) occurs mostly on north 
slopes. Staghorn cholla (Opuntia versicolor), prickly pear (O. phaeacantha), and fishhook barrel cactus (Ferocactus wislizenii) are scattered throughout. Summer rains support a diverse grass flora. The dominant species are sideoats grama (Bouteloua curtipendula), slender grama (B. repens), curly mesquite (Hilaria belangeri), tanglehead (Heteropogon contortus), and spidergrass (Aristida ternipes).

\section{Desert Riparian}

This vegetation type is found in the main drainage, Wildhorse Canyon, and the secondary drainages to the south at approximately 940-1220 m (3100-4000 ft) elevation. In places bush muhly (Muhlenbergia rigens) carpets the bottom of the canyon. Dominant woody plants include catclaw acacia (Acacia greggii), buttonbush (Cephalanthus occidentalis), wait-a-minute bush (Mimosa biuncifera), desert hackberry (Celtis pallida), canyon ragweed (Ambrosia ambrosioides), desert honeysuckle (Anisacanthus thurberi), and mesquite (Prosopis velutina). Canyon grape (Vitis arizonica), seep willow (Baccharis glutinosa), Goodding willow (Salix gooddingii) and coyote willow (S. exigua) are locally common.

\section{Desert-grassland}

Desert-grassland occurs from approximately 1220 to $1400 \mathrm{~m} \mathrm{(4000} \mathrm{to} 4600 \mathrm{ft}$ ). This variable community intergrades with the saguaro-paloverde desertscrub at its lower edge and the juniperoak woodland at its upper edge. It is characterized by the presence of numerous perennial grasses which flower largely in response to summer rains. The dominant native grasses are bull grass (Muhlenbergia emersleyi), sideoats grama (Bouteloua curtipendula), hairy grama (B. hirsuta), slender grama (B. repens), tanglehead (Heteropogon contortus), plains lovegrass (Eragrostis intermedia), and cane beardgrass (Bothriochloa barbinodis). Ocotillo, turpentine bush, silver indigo bush, (Dalea pulchra), and shindagger (Agave schotti) are common shrubs. Shindagger forms dense stands in places. Other common but scattered plants are sotol (Dasylirion wheeleri), pine needle milkweed (Asclepias linaria), Arizona rosewood (Vauquelinia californica), mesquite, wild cotton (Gossypium thurberi), staghorn cholla, and prickly pear cactus.

\section{Juniper-Oak Woodland}

Juniper-oak woodland is found only on north-facing slopes at the highest elevations in the extreme southeast corner of the PNA (1340-1400 m/4400-4600 ft). These slopes support an open grassy woodland. The scattered trees include alligator bark juniper (Juniperus deppeana), Emory oak (Quercus emoryi), and Mexican blue oak (Q. oblongifolia) with an occasional pinyon pine (Pinus discolor). The dominant shrubs are bear grass (Nolina microcarpa), manzanita (Arctostaphylos pungens), and shindagger. A diverse mixed grass understory is dominated by bullgrass and Texas beardgrass (Schizachrium cirratum). The dominant forbs, concentrated on bedrock benches, are spike moss (Selaginella rupincola) and bearded lip fern (Cheilanthes wootoni).

\section{Introduced Species}

Twenty-two introduced species, of which half are grasses, were found in the PNA. They constitute $6 \%$ of the local flora and are denoted in the checklist with an asterisk $(*)$. Several of these were common or are known to be especially invasive. Riparian ecosystems are fragile and extremely 
susceptible to invasions of exotic plants. Virtually all of the introduced species were found in the main canyon or its side drainages. Some of these also extended up the slopes.

The introduced flora in Wildhorse Canyon varied dramatically from spring to fall as several very common annuals flourished seasonally. Red brome (Bromus madritensis) is an opportunistic annual that was abundant throughout the site in the spring due to ample winter rains. It formed dense stands in the lower part of the canyon both above and below the tinaja. Bedstraw (Galium aparine), another spring annual, was also abundant in the canyon bottom, particularly along the shady, north-facing wall. In September these species were gone, replaced primarily by common cocklebur (Xanthium strumarium), a cosmopolitan summer annual of questionable origin, which formed dense thickets in the canyon bottom extending as far up as $1100 \mathrm{~m}(3600 \mathrm{ft})$.

Introduced perennial species were mostly grasses. Several patches of fountain grass (Pennisetum setaceum) were observed in the canyon and at least one side drainage. It is an escapee from cultivation and easily invades watercourses, roadways and occasionally hillsides. We expect that it will flourish in the Rincons as it has done elsewhere if measures are not taken to eradicate it. Natal grass (Rhynchelytrum repens) was found scattered throughout the PNA, although it was denser in the canyon bottom. It is not nearly as invasive as fountain grass. Lehmann lovegrass (Eragrostis lehmanniana), a summer flowering species which spreads rapidly, particularly in disturbed areas, was scattered. It was, however, abundant along Wildhorse trail just north of the PNA. Bermuda grass (Cynodon dactylon) was locally common in patches in the canyon bottom, especially in the vicinity of the tinaja. It is a tenacious grass that does well in any mesic, disturbed situation.

Only a few individuals of the strongly invasive tamarisk (Tamarix ramosissima) were found at $1100 \mathrm{~m}(3600 \mathrm{ft})$ in the canyon bottom. This tree is notorious for spreading along both perennial and intermittent streams in the southwest U.S. and could do well in Wildhorse Canyon. It, too, should be targeted for eradication before it becomes a real problem. The other exotic species were relatively uncommon and none pose a threat to the native vegetation.

\section{Additions to the Flora}

Nine species which are denoted in the checklist with a plus sign $(+)$ are new to the Rincon Flora (Bowers and McLaughlin 1987). Five are natives: maidenhair fern (Adiantum capillus-veneris), Parish Indian mallow (Abutilon parishii), Sonoran bursage (Ambrosia cordifolia), stickleaf (Mentzelia affinis), and red spiderling (Boerhavia gracillima). Maidenhair fern is found in Wildhorse Canyon below shady moist overhangs. Two populations were discovered at $975 \mathrm{~m}$ $(3200 \mathrm{ft})$ and $1050 \mathrm{~m}(3450 \mathrm{ft})$ in the main canyon. Parish Indian mallow is a summer-flowering perennial herb found among boulders and rock shelves on slopes above the canyon bottom. It is scattered throughout in small numbers. A few shrubs of Sonoran bursage were found among the abundant canyon ragweed in the streambed. These plants were atypical and appeared to be introgressed with the canyon ragweed based on examination of the leaf and fruit morphology. Stickleaf, a spring annual, was locally common in the canyon bottom, especially below the tinaja. Red spiderling, a summer-flowering perennial herb, was uncommon on a west-facing slope in saguaro-paloverde desertscrub. 
Three of the introduced species new to the flora are escapes from cultivation: African daisy (Dimorphotheca sinuata), a spring annual, was rare (one individual) on the north slope above Wildhorse Canyon. Fountain grass was occasional in the main canyon and a side drainage below the tinaja. A single individual of pampas grass (Cortaderia sellowana) was observed at approximately $1100 \mathrm{~m}(3600 \mathrm{ft})$ in a very steep part of the canyon. These three exotics are commonly grown ornamentals in the Tucson area. In contrast, weeping lovegrass (Eragrostis curvula), a perennial native to Africa, was deliberately introduced by the Soil Conservation Service as a possible range forage grass (Gould 1977). It has since spread throughout the southwest. In Wildhorse Canyon a small population was found only in one narrow side drainage.

\section{Sensitive Species}

Parish Indian mallow (Abutilon parishii) has a widely scattered distribution in the lower canyons of the Santa Catalina Mountains and is known from a few areas in the Tucson Mountains. The Wildhorse Canyon record is the first for the species from the Rincons. The plant, designated a C2 species before that protection category disappeared, is not considered threatened or endangered at this time. The $\mathrm{C} 2$ label (candidate for listing) indicated decline and that more information was needed about the species. On return visits in May 1992, Van Devender and Bertelsen located 63 individuals from 7 sites in both the canyon and its main side drainage, the largest number of plants known to date from a single area. The largest populations were found on southwest-facing rock shelves below the tinaja at about $960 \mathrm{~m}$ (3150 ft) elevation (13 plants) and on a shady, eastfacing canyon wall at about $1000 \mathrm{~m}$ (3200 ft) elevation (19 plants).

\section{Management and Protection Needs}

Wildhorse Canyon and its side drainages and slopes support a rich flora. In spite of the small area (approximately $194 \mathrm{ha} / 420 \mathrm{ac}$ ), over one-third of all species (360/986) found in the Rincon Mountains are represented in the Wildhorse PNA. The streambed is an unusual riparian corridor in a desert biome. Water remains throughout the canyon in potholes and basins year-round; it supports aquatic wildlife as well as provides a drinking source for terrestrial animals.

Except for the tinaja area, the PNA is seldom visited by people. Because the trail parallels or crosses the lower streambed several times, and the tinaja is a destination point for hikers and horseback riders, the potential for introducing exotic species is high. The riparian flora in and around the tinaja is strongly altered. As the canyon narrows and the gradient increases upstream introduced species become less common. Intensive seasonal floods provide a natural barrier to plant dispersal. Given enough time, however, exotics may migrate up the entire length of Wildhorse Canyon.

Fountain grass was not known to occur in the Rincon Mountains prior to publication of the Bowers and McLaughlin (1987) flora, but small populations are established in lower Wildhorse Canyon. We predict rapid increase of this species since we have seen explosive invasions in similar canyons in the Santa Catalina and the Tucson Mountains. Pima County Parks and Recreation Department has had some success controlling fountain grass by physically digging up plants along Kinney Road in the Tucson Mountains. 
Tamarisk is so scarce in Wildhorse Canyon that it could be easily eradicated. Once it becomes well established little can be done to control this noxious tree that can out-compete all other riparian species.

Common cocklebur is a stout annual that sprouts in response to summer rains. The woody, hooked fruits are easily transported on clothing and animal hair. However, it cannot survive on dry slopes and thus is limited to larger drainages. It might be possible to have mid-summer weeding sessions while the plants are still small and fruits are not ripe. We expect such an effort would have to be repeated for several years to have a significant effect.

Natal grass and Lehmann lovegrass are so scattered and widespread that it will probably take serious research to determine an effective method of elimination. The annual red brome is an even more difficult problem. It seeds prolifically and is abundant in wet years in all habitats. Control of exotics is always a difficult chore that requires constant vigilance. We recommend that the park consult a specialist in eradication of introduced species. It will also be necessary to limit access to the tinaja, especially by horses, in order to reduce the immigration and migration of exotics. We recommend that Wildhorse Canyon be maintained as a Protected Natural Area with use restrictions that could stem the tide of introduced species up the canyon. If such measures are taken, this canyon will continue to be a rare and outstanding habitat in the Rincon Mountains. Otherwise, it will inevitably become a less interesting and less diverse ecosystem. 
Table 5. Wildhorse Canyon Checklist of Vascular Plants. Wildhorse Canyon is a Protected Natural Area within the Rincon Mountain District of Saguaro National Park. This list was compiled in 1992 by Renée Rondeau and Rebecca Van Devender. Plant names follow Kartesz (1994).

$+=$ New addition to the flora of the Rincon Mountains of Bowers and McLaughlin (1987)

$*=$ Introduced species

\section{Acanthaceae}

Anisacanthus thurberi (Torrey) Gray

Carlowrightia arizonica Gray

Siphonoglossa longiflora (Torrey) Gray

\section{Adiantaceae}

Adiantum capillus-veneris L.

Bommeria hispida (Mett.) Underw.

Cheilanthes bonariensis (Willd.) Proctor

Cheilanthes lindheimeri Hook.

Cheilanthes wootonii Maxon.

Cheilanthes wrightii (Hooker)

Astrolepis sinuata (Lag. ex Sw) Bentham \&

Windham

ssp. sinuata

Notholaena standleyi Maxon

Pellaea truncata Goodding

Pellaea wrightiana (Hook.)

Pityrogramma triangularis (Kaulf.) Maxon

\section{Agavaceae}

Agave cf. palmeri Engelmann

Agave schottii Engelmann var. schottii

Yucca baccata Torrey var. brevifolia (Schott

ex Torr. )

L. Benson \& Darrow

\section{Amaranthaceae}

Amaranthus palmeri S. Watson

Froelichia arizonica Thornber ex Standley

Iresine heterophylla Standl.

\section{Apiaceae}

Bowlesia incana Ruiz \& Pav.

Daucus pusillus Michx.
Lomatium nevadense (S. Watson) Coulter \& Rose

Spermolepis echinata (Nuttall) Heller

\section{Apocynaceae}

Haplophyton crooksii L.

\section{Aristolochiaceae}

Aristolochia watsonii Wooton \& Standley

Asclepiadaceae

Asclepias linaria Cavanilles

Cynanchum arizonicum (Gray) Shinners

Matelea arizonica (Gray) Shinners

Sarcostemma cynanchoides Decaisne

ssp. hartwegii (Vail) R. Holm

\section{Asteraceae}

Acourtia wrightii (Gray) Reveal \& King Ambrosia ambrosioides (Cavanilles) Payne

Ambrosia confertiflora DC.

+ Ambrosia cordifolia (Gray) Payne

Artemisia ludoviciana Nuttall

Antheropeas lanosum (Gray) Rydberg

[Eriophyllum lanosum (Gray) Gray]

Baccharis salicifolia (R. \& P.) Persoon

[B. glutinosa Persoon]

Baccharis sarothroides A. Gray

Bahia absinthifolia Bentham

Bahia dissecta (Gray) Britt.

Bidens aurea (Ait.) Sherff

Brickellia amplexicaulis B. L. Rob.

Brickellia californica (Torrey \& Gray) Gray

Brickellia coulteri Gray

Brickellia venosa (Wooton \& Standley) B.L.

Robinson 
Calycoseris wrightii Gray

Carphochaete bigelovii Gray

Cirsium neomexicanum Gray

Conyza canadensis (L.) Cronquist

Coreocarpus arizonicus (Gray) Blake

Dyssodia porophylloides Gray

Encelia farinosa Gray

Ericameria laricifolia (Gray) Shinners

Erigeron colomexicanus (A. Nels.)

Erigeron oreophilus Greenman

Eupatorium solidaginifolium Gray

Gnaphalium chilense Sprengel

Gnaphalium wrightii A. Gray

Gymnosperma glutinosum (Sprengel)

Lessing

Heterotheca subaxillaris (Lamarck) Britton

\& Rusby

Hymenothrix wrightii A. Gray

Isocoma tenuisecta Greene

*Lactuca serriola L.

Machaeranthera pinnatifida (Hooker)

Shinners

Machaeranthera tagetina Greene

Machaeranthera tephrodes A. Gray

Malacothrix clevelandii Gray

Microseris lindleyi (DC.) A. Gray

+*Osteospermum sinuatum (DC.) Norf.

Porophyllum gracile Bentham

Psilostrophe cooperi (A. Gray) Greene

Rafinesquia neomexicana Gray

Sanvitalia abertii Gray

Senecio douglasii DC. var. monoensis

(Greene) Jepson

Senecio lemmoni A. Gray

Senecio neomexicanus Gray

*Sonchus asper (L.) Hill

*Sonchus oleraceus L.

Stephanomeria pauciflora (Torrey) A.

Nelson

Stylocline micropoides Gray

Thymophylla pentachaeta (DC.) Small

var. pentachaeta

Trixis californica Kellog

Viguiera annua (Jones) Blake

Viguiera dentata (Cavanilles) Sprengel var. lancifolia Blake

*Xanthium strumarium L.

Zinnia acerosa (DC.) A. Gray

Bignoniaceae

Tecoma stans (L.) Juss. ex Kunth

\section{Boraginaceae}

Amsinckia intermedia Fisch. \& Mey.

Cryptantha barbigera (A. Gray) Greene

Cryptantha pterocarya (Torrey) Greene

Harpagonella palmeri Gray

Pectocarya platycarpa (Munz \& I. M. Johnston)

Munz \& I. M. Johnston

Pectocarya recurvata I. M. Johnston

Plagiobothrys collinus (Phil.) I. M. Johnst.

\section{Brassicaceae}

Arabis perennans S. Watson

*Capsella bursa-pastoris (L.) Medik.

Descurainia pinnata (Walt.) Bentham

Lepidium lasiocarpum Nuttall

Lepidium virginicum $\mathrm{L}$.

var. medium (Greene) C. L. Hitchcock

[L. medium Greene]

*Sisymbrium irio L.

Schoencrambe linearifolia (Gray) Rollins

Thysanocarpus elegans Fisch. \& Mey.

\section{Cactaceae}

Carnegia gigantea (Engelmann) Britton \& Rose

Coryphantha vivipara (Nuttall) Britton \& Rose

var. bisbeeana (Orcutt) L. Benson

Echinocereus fasciculatus (Engelmann) L.

Benson

Echinocereus fendleri (Engelmann)

Engelmann

ex. Rumpler var. rectispinus (Peebles) L.

Benson

Ferocactus wislizenii (Engelmann) Britton \&

Rose

Mammillaria heyderi Muhl. 
var. macdougalii (Rose) L. Benson

Mammillaria grahamii Engelmann var. grahamii

Opuntia bigelovii Engelmann

Opuntia chlorotica Engelmann \& Bigelow

Opuntia fulgida Engelmann

var. mammillata (Schott) Coulter

Opuntia leptocaulis DC.

Opuntia phaeacantha Engelmann

Opuntia phaeacantha Engelmann

var. laevis (Coulter) L. Benson

Opuntia spinosior (Engelmann) Toumey

Opuntia versicolor Engelmann

\section{Campanulaceae}

Triodanis holzingeri McVaugh

Triodanis perfoliata (L.) Nieuwland var. biflora (Ruiz \& Pavon) DC.

\section{Capparidaceae}

Polanisia dodecandra (L.) DC. ssp. tracysperma (Torrey \& Gray) Iltis

\section{Caryophyllaceae}

Cerastium gracile Dufour

Silene antirrhina $\mathrm{L}$.

\section{Chenopodiaceae}

Atriplex canescens (Pursh) Nuttall

Chenopodium graveolens Willd.

Chenopodium palmeri Standley

\section{Commelinaceae}

Commelina erecta $\mathrm{L}$.

Tradescantia occidentalis (Britton) Smythe

\section{Convolvulaceae}

Evolvulus alsinoides (L.) L.

Evolvulus arizonicus A. Gray

Ipomoea coccinea $\mathrm{L}$.

Ipomoea costellata Torrey

Ipomoea hederacea (L.) Jacq.

Ipomoea leptotoma (Torrey)

Jacquemontia pringlei A. Gray

\section{Crassulaceae}

Crassula connata var. connata

Graptopetalum rusbyi (Greene) Rose

\section{Crossosomataceae}

Crossosoma bigelovii S. Watson

\section{Cupressaceae}

Juniperus deppeana Steudel

\section{Cyperaceae}

Cyperus squarosis L.

* Cyperus esculentus L.

Cyperus fendlerianus Boeckl.

Eleocharis montevidensis Kunth.

\section{Ericaceae}

Arctostaphylos pungens H. B. K.

\section{Euphorbiaceae}

Acalypha neomexicana Mueller-Argoviensis

Chamaesyce capitellata Engelmann)

Millspaugh

Chamaesyce florida (Engelmann) Millspaugh

Chamaesyce hyssopifolia (L.) Standley

Chamaesyce melanadenia (Torrey)

Millspaugh

Euphorbia heterophylla L.

Jatropha cardiophylla (Torrey) Mueller-

Argoviensis

Tragia nepetifolia Cavanilles

\section{Fabaceae}

Acacia angustissima (Millspaugh) Kuntze

Acacia constricta Bentham

Acacia greggii Gray

Amorpha fruticosa L.

Astragalus nuttallianus DC.

Calliandra eriophylla Bentham

Dalea pringlei Gray

Dalea pulchra Gentry 
Dalea versicolor Zucc.

ssp. versicolor var. sessilis (Gray)

Barneby

Desmodium cf. batocaulon Gray

Desmodium rosei Schubert

Galactia wrightii Gray

Lotus humistratus Greene

Lotus oroboides (Kunth) Ottley var. oroboides

Lotus rigidus (Bentham) Greene

Lupinus concinnus Agardh.

Lupinus sparsiflorus Bentham

Macroptilium gibbosifolium (Ortega) A. Delgado

Marina parryi (Torrey \& Gray) Barneby

Mimosa aculeaticarpa Ortega var. biuncifera (Bentham) Barneby

Nissolia schottii (Torrey) Gray

Parkinsonia florida (Bentham ex Gray) Watson

Parkinsonia microphylla Torrey

Phaseolus acutifolius Gray

Prosopis velutina Wooton

Senna covesii (Gray) Irwin \& Barneby

Tephrosia tenella Gray

Vicia ludoviciana Nuttall

\section{Fagaceae}

Quercus emoryi Torrey

Quercus oblongifolia Torrey

\section{Fouquieriaceae}

Fouquieria splendens Engelmann ssp. splendens

\section{Garryaceae}

Garrya wrightii Torrey

Gentianaceae

Centaurium calycosum (Buckl.) Fern.

\section{Hydrophyllaceae}

Emmenanthe penduliflora Bentham

Eucrypta chrysanthemifolia (Bentham) Greene
Phacelia bombycina Wooton \& Standley

Phacelia coerulea Greene

Phacelia cryptantha Greene

Phacelia distans Bentham

Phacelia ramosissima Dougl. ex Lehm.

\section{Juncaceae}

Juncus bufonius L.

Juncus interior Wieg.

Juncus marginatus Rostk.

\section{Lamiaceae}

Hedeoma nana (Torrey) Briquet

Hyptis emoryi Torrey

Stachys coccinea Jacquin

\section{Liliaceae}

Calochortus ambiguus (M. E. Jones)

Ownbey

Calochortus kennedyi Porter

Dichelostemma pulchellum (Salisb.) Heller

\section{Loasaceae}

+ Mentzelia affinis Greene

Mentzelia albicaulis (Douglas) Torrey \& Gray

Mentzelia asperula Wooton \& Standley

\section{Loranthaceae}

Phoradendron californicum Nuttall

Phoradendron capitellatum Torrey

Phoradendron villosum (Nuttall) Nuttall

ssp. coryae (Trel.) Wiens

\section{Malpighiaceae}

Janusia gracilis A. Gray

\section{Malvaceae}

Abutilon abutiloides (Jacquin) Garcke

Abutilon incanum (Link.) Sweet

+*Abutilon parishii Watson

Gossypium thurberi Todaro

Herissantia crispa (L.) Brizicky

Hibiscus biseptus $\mathrm{S}$. Wats.

Hibiscus coulteri Harvey 
Sphaeralcea emoryi Torrey

Sphaeralcea fendleri Gray

Sphaeralcea laxa Wooton \& Standley

Moraceae

Morus microphylla Buckley

\section{Nolinaceae}

Dasylirion wheeleri S. Watson

Nolina microcarpa $\mathrm{S}$. Watson

\section{Nyctaginaceae}

Allionia incarnata L.

Boerhavia diffusa L. var. diffusa

Boerhavia erecta L.

+Boerhavia gracillima Heimerl.

Boerhavia intermedia M. E. Jones

Boerhavia scandens L.

Mirabilis coccineus (Torrey) Bentham \& Hooker

\section{Onagraceae}

Camissonia californica (Nuttall ex Torrey \& Gray) Raven

Epilobium canum (Greene) Raven ssp. latifolium (Hooker) Raven

Oenothera primiveris Gray

\section{Papaveraceae}

Eschscholtzia californica Cham. ssp. mexicana (Greene) C. Clark

Platystemon californicus Bentham

\section{Phytolaccaceae}

Rivina humilis L.

\section{Pinaceae}

Pinus cembroides Zuccarini

\section{Plantaginaceae}

Plantago insularis Eastw.

Plantago patagonica Jacquin

Plantago virginica $\mathrm{L}$.
Plumbaginaceae

Plumbago scandens L.

Poaceae

Aristida scabra Willd.

Aristida adscencionis L.

Aristida hamulosa Henr.

Aristida parishii Hitchcock.

Aristida ternipes Cavanilles

Bothriochloa barbinodis (Lagasca) Herter

Bouteloua aristidoides (Kunth) Grisebach

Bouteloua chondrosoides (Kunth) Griesbach

Bouteloua curtipendula (Michaux) Torrey

Bouteloua eripoda (Torrey) Torrey

Bouteloua hirsuta Lagasca

Bouteloua radicosa (Fourn.) Griffiths

Bouteloua repens (Kunth) Scribner \& Merrill

Bromus carinatus Hooker \& Arnott

*Bromus madritensis L.

Chloris virgata Swartz

$+{ }^{*}$ Cortaderia selloana (JA \& JH Schultes)

Asch. \& Graebn.

Cottea pappophoroides Kunth

Critesion murinum (L.) Love

ssp. leporinum (Link) Love

Critesion pusillum (Nuttall) Love

*Cynodon dactylon (L.) Persoon

Digitaria californica Henrard

Diplachne dubia (Kunth) Scribn.

Echinochloa colonum (L.) Link

Elymus elymoides (Rafinesque) Swezey

Enneapogon desvauxii P. Beauvois

*Eragrostis cilianensis (Allard)

Vignolo-Lutati ex Janchen

+*Eragrostis curvula (Schrad.) Nees

Eragrostis intermedia Hitchcock

*Eragrostis lehmanniana Nees

Eragrostis pectinacea (Michaux) Nees

Eriochloa aristida Vasey

Eriochloa lemmonii Vasey \& Scribner var. gracilis (Fourn.) Gould

Erioneuron pulchellum (Kunth) Tateoka

Heteropogon contortus (L.) Beauvois ex Roemer \& Schultes

Hilaria belangeri (Steud.) Nash 
Leptochloa filiformis (Lamarck) P. Beauvois Lycurus setosus (Nuttall) C. Reeder

Muhlenbergia arizonica Scribner

Muhlenbergia dumosa Scribner

Muhlenbergia emersleyi Vasey

Muhlenbergia microsperma (DC.) Kunth

Muhlenbergia monticola Buckley

Muhlenbergia porteri Scribner

Muhlenbergia rigens (Bentham) Hitchcock

Muhlenbergia sinuosa Swallen

Panicum bulbosum Kunth

Panicum hirticaule Presl.

+*Pennisetum setaceum (Forskal) Chiov.

*Phalaris canariensis L.

Poa bigelovii Vasey \& Scribner

*Polypogon monspeliensis (L.) Desfontaines

*Rhynchelytrum repens (Willdenow) C.E.

Hubbard

Schizachyrium cirratum (Hackel) Wooton \&

Standley

Schizachyrium sanguineum (Retzius) Alston

Setaria macrostachya H. B. K.

Sporobolus wrightii Munro

Tridens muticus (Torrey) Nash

Vulpia microstachys (Nuttall) Munro

Vulpia octoflora (Walter) Rydberg

Polemoniaceae

Eriastrum diffusum (A. Gray) Mason

Gilia flavocincta A. Nels.

ssp. australis (A. \& V. Grant) Day \&

Grant

Linanthus bigelovii (Gray) Greene

Microsteris gracilis (Hook.) Greene

\section{Polygonaceae}

Eriogonum abertianum Torrey

Eriogonum wrightii Torrey

Pterostegia drymarioides Fisch. \& Mey.

Rumex hymenosepalus Torrey

\section{Portulacaceae}

Calandrinia ciliata (Ruiz \& Pavon) DC.

Claytonia perfoliata Donn

Portulaca suffrutescens Engelmann
Talinum paniculatum (Jacquin) Gaertner

Primulaceae

Androsace occidentalis Pursch

Ranunculaceae

Anemone tuberosa Rydberg

Delphinium scaposum Greene

Myosurus culpulatus S. Watson

Rhamnaceae

Rhamnus crocea Nuttall ssp. ilicifolia (Kellogg) C.B. Wolf

Ziziphus obtusifolia (Hooker) Gray

var. canescens (Gray) M. C. Johnston

Rosaceae

Vauquelinia californica (Torrey) Sargent

Rubiaceae

Bouvardia ternifolia (Cavanilles)

Schlechtendal

Cephalanthus accidentalis L.

*Galium aparine L.

Galium microphyllum Gray

Galium proliferum Gray

Salicaceae

Populus fremontii S. Watson

Salix exigua Nuttall

Salix gooddingii Ball

Salix taxifolia Kunth

Santalaceae

Comandra umbellata (L.) Nuttall

ssp. pallida (DC.) Piehl

Sapindaceae

Dodonea viscosa (L.) Jacquin

Sapindus drummondii Hooker \& Arnott

\section{Saxifragaceae}

Fendleria rupicola Gray

Heuchera sanguinea Engelmann 


\section{Scrophulariaceae}

Antirrhinum nuttallianum Bentham

Castilleja tenuiflora Bentham

Linaria texana Scheele

Maurandya antirrhiniflora Humboldt \&

Bonpland

Mecardonia vandellioides (H. B. K.) Pennell

Mimulus guttatus DC.

Mimulus nasutus Greene

Penstemon barbatus (Cavanilles) Roth

Penstemon parryi (A. Gray) A. Gray

Stemodia durantifolia (L.) Swartz

Selaginellaceae

Selaginella arizonica Maxon

Selaginella rupincola Underw.

\section{Solanaceae}

Lycium berlandieri Dunal

Lycium exsertum A. Gray

Nicotiana trigonophylla Dunal

Physalis crassifolia Bentham

Solanum douglasii Dunal

\section{Sterculiaceae}

Ayenia compacta Rose

\section{Tamaricaceae}

*Tamarix ramosissima Ledeb.

\section{Typhaceae}

Typha domingensis Persoon

\section{Ulmaceae}

Celtis pallida Torrey

\section{Urticaceae}

Parietaria hespera Hinton

\section{Valerianaceae}

Plectritis ciliosa (Greene) Jepson ssp. insignis (Suksdorff) Morey

\section{Verbenaceae}

Aloysia wrightii A.A. Heller ex Abrams

Glandularia bipinnatifida (Nuttall) Nuttall var. bipinnatifida

\section{Vitaceae}

Vitis arizonica Engelmann 


\section{Literature Cited}

Bowers, J. E., and S. P. McLaughlin. 1987. Flora and vegetation of the Rincon Mountains, Pima County, Arizona. Desert Plants 8:51-95.

Gould, F. W. 1977. Grasses of Southwestern United States. University of Arizona Press, Tucson. $352 \mathrm{p}$.

Kartesz, J. T. 1994. A synonomized checklist of the vascular flora of the United States, Canada, and Greenland: second edition, 2-volume checklist/thesaurus. Biota of North America Program of the North Carolina Botanic Garden. Timber Press, Portland, Oregon. vol. 1, 622 p; vol. 2, 816 p.

National Park Service. 1988. Draft Final General Management Plan: Saguaro National Monument. Department of the Interior, National Park Service. Saguaro National Monument, Tucson, Arizona. 27 p.

Rondeau, R. J. 1991. Flora and vegetation of the Tucson Mountains, Pima County, Arizona. Master's thesis, University of Arizona, Tucson. 
The cover photograph was taken October 4, 1935, in Saguaro National Monument by the first National Park Service photographer, George Alexander Grant (1891-1964).

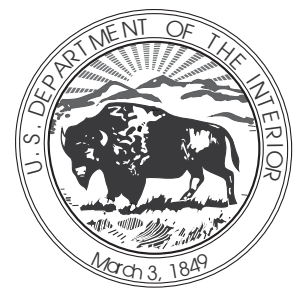

As the nation's principal conservation agency, the U.S. Department of the Interior has responsibility for most of our nationally owned public lands and natural and cultural resources. This includes fostering wise use of our land and water resources, protecting fish, wildlife and plants, preserving the environmental and cultural values of national parks and historic places, and providing for enjoyment of life through outdoor recreation. The Department assesses our energy and mineral resources and works to ensure that their development is in the best interests of all our people. The Department also has a major responsibility for American Indian reservation communities and for people who live in island territories under U.S. administration. 